\title{
An action principle for the masses of Dirac particles
}

\author{
Felix Finster and Stefan Hoch
}
Fakultät Mathematik, Universität Regensburg, 93040 Regensburg, Germany Felix.Finster@mathematik.uni-regensburg.de, Stefan.Hoch@mathematik.uni-regensburg.de

\begin{abstract}
A variational principle is introduced which minimizes an action formulated for configurations of vacuum Dirac seas. The action is analyzed in position and momentum space. We relate the corresponding EulerLagrange equations to the notion of state stability. Examples of numerical minimizers are constructed and discussed.
\end{abstract}

\section{Contents}

1 Introduction

2 A variational principle for Dirac sea configurations

3 A Plancherel formula for Lorentz invariant causal functions

4 The variational principle in momentum space, connection to state stability

e-print archive: http://lanl.arXiv.org/abs/0712.0678 
5 Convolutions of Lorentz invariant distributions

5.1 Convolutions of negative distributions

1677

5.2 Mixed convolutions

1679

5.3 Convolutions involving Dirac seas

1692

6 A Lorentz invariant regularization and its fourier transform

1697

7 Additional free parameters

1705

8 Numerical construction of minimizers

1706

Acknowledgment

1710

References

1710

\section{Introduction}

In the standard model of elementary particle physics many structures and parameters are built in ad hoc. In particular, the theory makes no statement on the masses of the leptons and quarks; the mass parameters need to be put in empirically from experimental data. This situation is not quite satisfying, and one would hope for a more fundamental explanation for the ratios of these masses. As an approach towards a theory which makes predictions for the mass parameters, we here set up a variational principle where we minimize an action formulated for configurations of vacuum Dirac seas.

The minimization problem is nonlocal, nonlinear and involves two or four free parameters. Therefore, a systematic study of the structure of all minimizers goes beyond the scope of this paper. But we construct and discuss numerical examples of minimizers. The technical core of the paper is to compute the Fourier transform of the action and to relate the regularization on the light cone to a suitable regularization procedure in momentum space. This transformation to momentum space simplifies the numerical analysis. Furthermore, it reveals a connection between the corresponding Euler-Lagrange equations and the notion of state stability as introduced in $[1, \S 5.6]$ for vacuum Dirac sea configurations.

Since the action principle for vacuum Dirac seas seems of interest by itself, this paper is self-contained and easily accessible. Nevertheless, our action 
principle can be seen in a broader context as a modification of the variational principle introduced in [1] for the fermionic projector in discrete space-time (see also [2] or the review articles [3,4]). More specifically, we modify the action of [1] such as to make it finite in the Lorentz invariant setting, treating the singularities on the light cone by a suitable regularization procedure. Our method is complementary to that in [1, Chapter 4]: whereas in [1] the behavior of the singularities on the light cone is considered in the so-called continuum limit, we here disregard these singularities completely and concentrate instead on the regular contribution away from the light cone. Yet another method is used in [5], where the vacuum Dirac seas are regularized, and it is analyzed how the operator product $\mathcal{M P}$ behaves as the regularization is removed. These different approaches all complement each other and should eventually be combined to get a full understanding of the structure of the minimizers of our variational principle. For an overview of the different methods we refer to the review paper [6].

The paper is organized as follows. In Section 2 we set up our variational principle in position space and discuss the relation to the variational principle in discrete space-time as introduced in [1]. In Section 3 we prove Plancherel-type formulas for Lorentz invariant functions. These Plancherel formulas are basic for the subsequent reformulation of our variational principle in momentum space. In Section 4 we outline the basic method and explain the connection to state stability, whereas Sections 5 and 6 are devoted to the more technical and computational aspects. In Section 7 we introduce two additional free parameters, which arise in the context of state stability, and built them into our framework by introducing the socalled extended action. In Section 8 we outline our numerical methods and discuss a few examples of numerical minimizers and state stable Dirac sea configurations.

\section{A variational principle for Dirac sea configurations}

In this section we shall formulate a variational principle for Lorentz invariant Dirac sea configurations. For clarity and self-consistency, we proceed without referring to the variational principle in discrete space-time as introduced in [1]. The connection to the latter variational principle will be explained later in this section (see after Definition 2.2). We begin with a general ansatz for the fermionic projector of the vacuum involving an arbitrary number of generations $g \in \mathbb{N}$ of Dirac seas of masses $m_{1}, \ldots, m_{g} \in \mathbb{R}$, which are taken into account with weight factors $\rho_{1}, \ldots, \rho_{g} \geq 0$ (for a discussion of the weight factors see [5, Appendix A]). More precisely, we introduce the distribution 
in momentum space

$$
\hat{P}(k)=\sum_{\beta=1}^{g} \rho_{\beta}\left(\not k+m_{\beta}\right) \delta\left(k^{2}-m_{\beta}^{2}\right) \Theta\left(-k^{0}\right)
$$

and consider its Fourier transform to position space

$$
P(\xi)=\int \frac{d^{4} k}{(2 \pi)^{4}} \hat{P}(k) \mathrm{e}^{\mathrm{i} k \xi}
$$

Here $k \xi$ denotes the inner product of signature $(+---)$ in Minkowski space $M$.

We first define our action formally and give the analytic justification afterwards. For the upper and lower light cone and its boundary we use the notations

$$
\begin{gathered}
I^{\vee}=\left\{\xi \mid \xi^{2}>0, \xi^{0}>0\right\}, \quad I^{\wedge}=\left\{\xi \mid \xi^{2}>0, \xi^{0}<0\right\}, \\
L=\left\{\xi \mid \xi^{2}=0\right\}
\end{gathered}
$$

For any $\xi \in I^{\vee}$ we introduce the so-called closed chain $A$ and its trace-free part $A_{0}$ by

$$
A=P(\xi) P(\xi)^{*}, \quad A_{0}=A-\frac{1}{4} \operatorname{Tr}(A),
$$

where the star denotes the adjoint with respect to the indefinite inner product $\bar{\Psi} \Phi$ on the Dirac spinors (where $\bar{\Psi}=\Psi^{\dagger} \gamma^{0}$ is the adjoint spinor). Thus

$$
P(\xi)^{*} \equiv \gamma^{0} P(\xi)^{\dagger} \gamma^{0}
$$

where the dagger denotes the transposed complex conjugate matrix. Due to Lorentz invariance, $A_{0}$ can be written in the upper light cone as

$$
A_{0}=\frac{\$}{2} f\left(\xi^{2}\right) \quad \text { for } \xi \in I^{\vee},
$$

and the relation $A_{0}^{*}=A_{0}$ implies that the function $f$ is real. Hence the Lagrangian

$$
\mathcal{L}=\operatorname{Tr}\left(A_{0}^{2}\right)=\xi^{2} f\left(\xi^{2}\right)^{2}
$$


is non-negative and depends only on the positive parameter $z:=\xi^{2}$. Thus the integral

$$
\mathcal{S} \stackrel{\text { formally }}{=} \int_{0}^{\infty} \mathcal{L}(z) z d z
$$

formally defines a positive functional depending on the free parameters $m_{1}, \ldots, m_{g}$ and $\rho_{1}, \ldots, \rho_{g}$.

Let us now give definitions (2.3)-(2.6) a rigorous mathematical meaning. An explicit calculation of the Fourier transform (2.2) (see [5, Section 3]) shows that the distribution $P(\xi)$ has the following properties. First, it is singular on the light cone $L$, but is a smooth function otherwise. Furthermore, it is Lorentz invariant. This means in the upper light cone that $P(\xi)$ can be written as

$$
P(\xi)=\notin v\left(\xi^{2}\right)+h\left(\xi^{2}\right) \quad \text { for } \xi \in I^{\vee}
$$

with smooth complex functions $v, h \in C^{\infty}\left(\mathbb{R}^{+}\right)$, which can be given explicitly in terms of Bessel functions. Thus the pointwise computations (2.3) and (2.5) make sense and

$$
f(z)=\operatorname{Re}(v(z) \overline{h(z)}) \in C^{\infty}\left(\mathbb{R}^{+}\right) .
$$

We conclude that the integrand in (2.6) is a smooth function. Near infinity, an asymptotic expansion of the Bessel functions yields that the function $f$ decays like $\mathcal{O}\left(z^{-2}\right)$, and thus the integral in (2.6) is absolutely convergent at infinity. Near the origin, $f$ has the expansion (see [5, equation (3.5)])

$$
f(z)=\frac{\mathfrak{m}_{3}}{z^{2}}+\frac{\mathfrak{m}_{5}}{z}+\mathcal{O}(\log z)
$$

where we set

$$
\begin{aligned}
\mathfrak{m}_{3} & =-\frac{1}{64 \pi^{5}} \sum_{\alpha, \beta=1}^{g} \rho_{\alpha} \rho_{\beta}\left(m_{\alpha}^{3}+m_{\beta}^{3}\right), \\
\mathfrak{m}_{5} & =\frac{1}{512 \pi^{5}} \sum_{\alpha, \beta=1}^{g} \rho_{\alpha} \rho_{\beta}\left(m_{\alpha}-m_{\beta}\right)^{2}\left(m_{\alpha}+m_{\beta}\right)^{3} .
\end{aligned}
$$

Thus according to (2.5), the integrand in (2.6) has a nonintegrable pole at $z=0$,

$$
\operatorname{Tr}\left(A_{0}^{2}\right) z=f(z)^{2} z^{2}=\frac{\mathfrak{m}_{3}^{2}}{z^{2}}+\frac{2 \mathfrak{m}_{3} \mathfrak{m}_{5}}{z}+\mathcal{O}(\log z) .
$$

In order to make sense of the integral, we need to subtract suitable counter terms. The simplest method is to add indefinite integrals of the poles 
in (2.11) evaluated at $z=\varepsilon$,

$$
\lim _{\varepsilon \searrow 0}\left(\int_{\varepsilon}^{\infty} \operatorname{Tr}\left(A_{0}^{2}\right) z d z-\frac{\mathfrak{m}_{3}^{2}}{\varepsilon}+2 \mathfrak{m}_{3} \mathfrak{m}_{5} \log \varepsilon\right) .
$$

However, since the indefinite integrals are determined only up to constants, it seems natural to allow for adding arbitrary constants times $\mathfrak{m}_{3}$ and $\mathfrak{m}_{3} \mathfrak{m}_{5}$. This freedom of modifying the action will turn out to be very useful later. In order to be even more flexible, it is preferable to treat the freedom more generally by a function $F\left(\mathfrak{m}_{3}, \mathfrak{m}_{5}\right)$. This gives rise to the following definition.

Definition 2.1. (Lorentz invariant action). For any given function $F \in$ $C^{\infty}(\mathbb{R} \times \mathbb{R}, \mathbb{R})$ we define the action $\mathcal{S}=\mathcal{S}\left(m_{1}, \ldots, m_{g}, \rho_{1}, \ldots, \rho_{g}\right)$ by

$$
\mathcal{S}=\lim _{\varepsilon \searrow 0}\left(\int_{\varepsilon}^{\infty} \operatorname{Tr}\left(A_{0}^{2}\right) z d z-\frac{\mathfrak{m}_{3}^{2}}{\varepsilon}+2 \mathfrak{m}_{3} \mathfrak{m}_{5} \log \varepsilon\right)+F\left(\mathfrak{m}_{3}, \mathfrak{m}_{5}\right) .
$$

Here $A_{0}$ is defined by $(2.3)$ for any $\xi \in I^{\vee}$ and $z=\xi^{2}$. The parameters $\mathfrak{m}_{3}$ and $\mathfrak{m}_{5}$ are defined by (2.9) and (2.10).

Note that due to the negative counter terms, action (2.12) need no longer be positive. But for any fixed $\varepsilon>0$ it is clearly bounded from below. We remark that in Definition 7.1 we shall extend this action by additional summands involving two more free parameters.

Next, we want to introduce a corresponding variational principle. In order to avoid the trivial minimizers $\rho_{1}=\cdots=\rho_{g}=0$ or $m_{1}=\cdots=m_{g}=0$, we need to impose a constraint.

Definition 2.2. (Lorentz invariant variational principle). We minimize the action $\mathcal{S}$, (2.12), varying the parameters $\rho_{1}, \ldots, \rho_{g} \geq 0$ and $m_{1}, \ldots, m_{g} \geq 0$ under the constraint

$$
\mathcal{T}:=\sum_{\beta=1}^{g} \rho_{\beta} m_{\beta}^{3}=1
$$

In order to understand the relation between the above variational principle and the variational principle introduced in discrete space-time in $[1, \S 3.5,2]$, we first consider the Lagrangian (2.5) for vectors $\xi$ which are space-like or inside the past light cone $I^{\wedge}$. Taking the adjoint of (2.2) and (2.1), one sees that

$$
P(-\xi)=P(\xi)^{*}
$$

Hence the closed chain $(2.3)$ can be written as $A=P(\xi) P(-\xi)$. Due to ansatz (2.1) and (2.2), the matrices $P( \pm \xi)$ are of the form $\alpha_{ \pm} \not \dot{\phi}+\beta_{ \pm}$with 
$\alpha_{ \pm}, \beta_{ \pm} \in \mathbb{C}$. This shows in particular that the matrices $P(\xi)$ and $P(-\xi)$ commute, and thus

$$
A(-\xi)=A(\xi) \quad \text { and } \quad A_{0}(-\xi)=A_{0}(\xi)
$$

For space-like $\xi$, we can use Lorentz symmetry to write the matrix $A_{0}(\xi)$ in the form $A_{0}(\xi)=\$ g\left(\xi^{2}\right)$ with a function $g \in C^{\infty}((-\infty, 0))$. Replacing $\xi$ by $-\xi$ and using (2.14), we see that $g$ must vanish identically. Combining this with (2.4) and (2.14), we conclude that away from the light cone, $A_{0}$ is of the form

$$
A_{0}(\xi)=\frac{\$}{2} f\left(\xi^{2}\right) \Theta\left(\xi^{2}\right) \epsilon\left(\xi^{0}\right) \quad \text { if } \xi \notin L
$$

(where $\epsilon$ is the step function $\epsilon(x)=1$ if $x \geq 0$ and $\epsilon(x)=-1$ otherwise). In words, $A_{0}$ is reflection symmetric (2.14), causal and Lorentz invariant. Moreover, for time-like $\xi$ the roots $\lambda_{1}, \ldots, \lambda_{4}$ of the characteristic polynomial of $A$ (counted with multiplicities) are computed to be real. According to [5, Lemma 2.1], these roots all have the same sign. Combining these facts, we can write the Lagrangian (2.5) in the alternative form

$$
\mathcal{L}=\operatorname{Tr}\left(A^{2}\right)-\frac{1}{4} \operatorname{Tr}(A)^{2}=\left|A^{2}\right|-\frac{1}{4}|A|^{2} \quad \text { if } \xi^{2} \neq 0
$$

where $|$.$| is the spectral weight (see [1, \S 3.5]$ )

$$
|A|=\sum_{i=1}^{4}\left|\lambda_{i}\right| \text {. }
$$

This is precisely the Lagrangian in the "model example" introduced in [1, $\S 3.5]$, for a particular value of the Lagrange multiplier. The same Lagrangian also appears in [2] as the so-called critical case of the auxiliary variational principle. As this Lagrangian vanishes for space-like $\xi$ and is symmetric under the transformation $\xi \rightarrow-\xi$, it was no loss of generality in Definition 2.1 to restrict attention to $\xi$ in the upper light cone. Hence the only differences between the above variational principle and the variational principle in $[1,2]$ are the following points:

(i) A regularization on the light cone is used in (2.12).

(ii) The additional constraint (2.13) appears.

(iii) The method of summing or integrating over space-time is different. We can describe the transition from the action in $[1,2]$ to $(2.6)$ by the replacement

$$
\sum_{x, y \in M} \cdots \longrightarrow \int_{0}^{\infty} \cdots z d z
$$


Let us briefly discuss these points. It is not astounding that a regularization on the light cone is necessary, because the fermionic projector has poles and singularities on the light cone. Such regularizations have been used previously in the derivation of the continuum limit [1, Chapter 4] and for the vacuum fermionic projector in [5]. The new and pleasant feature of regularization (i) is that it does not break Lorentz invariance. The necessity of constraint (ii) can be understood as replacing the condition in discrete space-time that the number of particles is fixed. However, the detailed form of constraint (2.13) will not become clear before Section 4. Replacement (iii) requires a detailed explanation. The natural method to describe the transition from discrete to a continuum space-time is to replace sums by space-time integrals,

$$
\sum_{x, y \in M} \cdots \longrightarrow \int_{M} d^{4} x \int_{M} d^{4} y \cdots=\int_{M} d^{4} x \int_{M} d^{4} \xi \cdots
$$

where in the last step we made a change of variables. For homogeneous systems as considered here, where the fermionic projector depends only on the difference $y-x$, the integrand of the $x$-integral is a constant. Thus this integral merely gives an infinite constant, and it seems natural to simply drop it. Thus it remains to discuss the replacement

$$
\int_{M} \cdots d^{4} \xi \rightarrow \int_{0}^{\infty} \cdots z d z
$$

This replacement has no simple justification; it changes the variational principle substantially. The integral on the right has the nice property that it can be finite for Lorentz invariant integrands, whereas the left integral is necessarily infinite for Lorentz invariant integrands, because the integrals over the hyperbola $\xi^{2}=$ const diverge. Furthermore, the integration measure $z d z$ in (2.16) can be motivated from the fact that it has the same dimension of length four as the measure $d^{4} \xi$. However, we again point out that replacement (2.16) changes the variational principle completely. Thus the variational principle introduced here is not merely a "continuum version" of the variational principle in $[1,2]$. It is a different variational principle. Nevertheless, as we shall see in Section 4, there is a close connection between minimizers of the variational principle of Definition 2.2 and the notion of state stability as introduced in $[1, \S 5.6]$.

We close this section with the derivation of the corresponding EulerLagrange (EL) equations. We denote the first variation of the masses $m_{\beta}$ and weights $\rho_{\beta}$ by $\delta m_{\beta}$ and $\delta \rho_{\beta}$, respectively. We let $\delta P$ be the corresponding first variation of the fermionic projector (2.1) and (2.2). The variation of 
the Lagrangian is

$$
\delta \operatorname{Tr}\left(A_{0}^{2}\right)=2 \operatorname{Tr}\left(A_{0} \delta A_{0}\right)=2 \operatorname{Tr}\left(A_{0} \delta A\right)
$$

where in the last step we used that the scalar component of $\delta A$ drops out of the trace because the other factor $A_{0}$ only has a vector component. Using (2.3) and (2.14), we conclude that

$$
\begin{aligned}
\delta \operatorname{Tr}\left(A_{0}^{2}\right) & =\operatorname{Tr}\left(A_{0}\left(\delta P(\xi) P(\xi)^{*}+P(\xi) \delta P(\xi)^{*}\right)\right) \\
& =2 \operatorname{Re} \operatorname{Tr}\left(A_{0} P(\xi) \delta P(-\xi)\right)
\end{aligned}
$$

Treating the constraint with a Lagrange multiplier $\lambda$, the EL equations become

$$
\begin{aligned}
& \lim _{\varepsilon \searrow 0}\left(\int_{\varepsilon}^{\infty} \operatorname{Re} \operatorname{Tr}\left(A_{0} P(\xi) \delta P(-\xi)\right) z d z-\frac{\mathfrak{m}_{3} \delta \mathfrak{m}_{3}}{\varepsilon}\right. \\
& \left.\quad+\left(\delta \mathfrak{m}_{3} \mathfrak{m}_{5}+\mathfrak{m}_{3} \delta \mathfrak{m}_{5}\right) \log \varepsilon\right)+\delta F\left(\mathfrak{m}_{3}, \mathfrak{m}_{5}\right)-\lambda \delta \mathcal{T}=0
\end{aligned}
$$

This equation looks rather complicated, and in this form it does not seem helpful for understanding our variational principle. In order to clarify the situation, in the next sections we shall transform our variational principle to momentum space.

\section{A Plancherel formula for Lorentz invariant causal functions}

Studying the Lorentz invariant action (2.12) directly in position space has the following disadvantages. First, expressing $A_{0}$ explicitly using Bessel function, one sees that $A_{0}(z)$ is oscillatory for large $z$, making it difficult to analytically estimate or to numerically compute the integral in (2.12). Second, the corresponding EL equations in position space (2.17) have no obvious interpretation. For these reasons, it is preferable to transform the action (2.12) to momentum space. To this end, we need a Plancherel-type formula, which relates the integral over the position variable $z=\xi^{2}$ to an integral over a momentum variable $a:=k^{2}$. In this section we shall derive such Plancherel formulas. For clarity, we begin with scalar functions. Thus, 
leaving out the Dirac matrices in (2.15), we consider functions of the form

$$
F(\xi)=f\left(\xi^{2}\right) \Theta\left(\xi^{2}\right) \epsilon\left(\xi^{0}\right), \quad G(\xi)=g\left(\xi^{2}\right) \Theta\left(\xi^{2}\right) \epsilon\left(\xi^{0}\right)
$$

with measurable complex-valued functions $f$ and $g$. In view of the integration measure in (2.6), we introduce on such functions the inner product

$$
\langle F, G\rangle:=\int_{0}^{\infty} \overline{f(z)} g(z) z d z .
$$

We denote the space of functions for which the last integral converges absolutely, by $L^{2}(M, z d z)$. For the upper and lower mass cone and their union we introduce the notations

$$
\begin{aligned}
& \mathcal{C}^{\vee}=\left\{k \mid k^{2}>0, k^{0}>0\right\}, \quad \mathcal{C}^{\wedge}=\left\{k \mid k^{2}>0, k^{0}<0\right\} \\
& \mathcal{C}=\left\{k \mid k^{2}>0\right\}
\end{aligned}
$$

Taking the usual Fourier transform in Minkowski space

$$
\hat{F}(k)=\int d^{4} \xi F(\xi) \mathrm{e}^{-\mathrm{i} k \xi}
$$

a symmetry argument shows that $\hat{F}(-k)=-F(k)$, and from Lorentz invariance we conclude that $\operatorname{supp} \hat{F} \subset \overline{\mathcal{C}}$. Hence $\hat{F}$ can be written similar to (3.1) as

$$
\hat{F}(k)=\hat{f}\left(k^{2}\right) \Theta\left(k^{2}\right) \epsilon\left(k^{0}\right) .
$$

On such functions we introduce similar to (3.2) the inner product

$$
\langle\hat{F}, \hat{G}\rangle:=\frac{1}{(2 \pi)^{4}} \int_{0}^{\infty} \overline{\hat{f}(a)} \hat{g}(a) a d a,
$$

where we set $a=k^{2}$. The space of functions, for which the last integral converges absolutely, is denoted by $L^{2}(\hat{M}, a d a)$.

Theorem 3.1. (Lorentz invariant Plancherel formula, scalar case). For functions of the form (3.1), the Fourier transform (3.3) is a unitary mapping from $L^{2}(M, z d z)$ to $L^{2}(\hat{M}, a d a)$. In particular, for all $F, G \in$ $L^{2}(M, z d z)$,

$$
\int_{0}^{\infty} \overline{f(z)} g(z) z d z=\frac{1}{(2 \pi)^{4}} \int_{0}^{\infty} \overline{\hat{f}(a)} \hat{g}(a) a d a .
$$


Proof. A possible method would be to express the transformation from $f$ to $\hat{f}$ with Bessel functions, and to use properties of the so-called Hankel transformation (see for example $[9,10]$ ). In order to avoid working with special functions, we shall here use a different method, which also has the advantage of explaining the integration measures $z d z$ and $a d a$. Nevertheless, we briefly outline the connection to the Hankel transformation: Rewriting the Fourier integral (3.3) as

$$
\hat{F}(k)=\int_{0}^{\infty} f(z) d z \int d^{4} \xi \delta\left(\xi^{2}-z\right) \epsilon\left(\xi^{0}\right) \mathrm{e}^{-\mathrm{i} k \xi},
$$

we can carry out the last integral using Bessel functions. Comparing with (3.4) we obtain

$$
\hat{f}(a)=2 \mathrm{i} \pi^{2} \int_{0}^{\infty} f(z) a \frac{J_{1}(\sqrt{a z})}{\sqrt{a z}} d z .
$$

After transforming to the variables $x=\sqrt{z}$ and $y=\sqrt{a}$, we can apply Parseval's equation for the Hankel transform [10, Theorem 5.1.2].

We now give the alternative proof which avoids special functions. Using a standard approximation argument, it suffices to prove the theorem for functions $F$ and $G$ of the form (3.1) with smooth and compactly supported $f, g \in C_{0}^{\infty}\left(\mathbb{R}_{+}\right)$. A short calculation shows that then the wave operator becomes

$$
-\square F(\xi)=(W f)\left(\xi^{2}\right) \Theta\left(\xi^{2}\right) \epsilon\left(\xi^{0}\right),
$$

where $W$ is the ordinary differential operator

$$
(W f)(z)=-\frac{4}{z} \frac{d}{d z}\left(z^{2} \frac{d}{d z} f\right) .
$$

Integrating by parts, one sees that $W$ is a nonnegative symmetric operator on the Hilbert space $L^{2}\left(\mathbb{R}^{+}, z d z\right)$ with domain of definition $C_{0}^{\infty}\left(\mathbb{R}^{+}\right)$.

In order to construct a self-adjoint extension of $W$, we use the wellknown fact that the operator $-\partial_{z}^{2}$ is self-adjoint on $L^{2}\left(\mathbb{R}^{+}, d z\right)$ with domain $\mathcal{D}\left(-\partial_{z}^{2}\right)=H_{0}^{2,2}\left(\mathbb{R}^{+}\right)$. We set

$$
\mathcal{D}(W)=\left\{u \text { measurable with } z u(z) \in \mathcal{D}\left(-\partial_{z}^{2}\right)\right\} .
$$

To verify that $W$ is self-adjoint, we use the definition of self-adjointness. Suppose that for given $v, w \in L^{2}\left(\mathbb{R}^{+}, z d z\right)$,

$$
\langle W u, v\rangle_{L^{2}\left(\mathbb{R}^{+}, z d z\right)}=\langle u, w\rangle_{L^{2}\left(\mathbb{R}^{+}, z d z\right)} \quad \forall u \in \mathcal{D}(W) .
$$


A short calculation shows that

$$
W u=-\partial_{z}^{2} \tilde{u} \quad \text { with } \quad \tilde{u}(z):=z u(z)
$$

This allows us to rewrite (3.7) as

$$
\left\langle-\partial_{z}^{2} \tilde{u}, \tilde{v}\right\rangle_{L^{2}\left(\mathbb{R}^{+}, d z\right)}=\langle\tilde{u}, w\rangle_{L^{2}\left(\mathbb{R}^{+}, d z\right)} \quad \forall \tilde{u} \in \mathcal{D}\left(-\partial_{z}^{2}\right)
$$

Since the operator $\left(-\partial_{z}^{2}\right)$ is self-adjoint, it follows that $\tilde{v} \in \mathcal{D}\left(-\partial_{z}^{2}\right)$ and that $-\partial_{z}^{2} \tilde{v}=w$. Hence $v \in \mathcal{D}(W)$ and $W v=w$, showing that $W$ with domain (3.6) is indeed self-adjoint.

The spectral theorem yields

$$
\langle f, g\rangle_{L^{2}\left(\mathbb{R}^{+}, z d z\right)}=\int_{\sigma(A)}\left\langle f, d E_{a} g\right\rangle_{L^{2}\left(\mathbb{R}^{+}, z d z\right)}
$$

where $d E_{a}$ is the corresponding spectral measure. In order to get more information on the spectrum, it is most convenient to work in momentum space, where the operator $-\square$, and consequently also the operator $W$, coincide with the multiplication operator $\hat{f}(k) \mapsto k^{2} \hat{f}(k)$. Then it becomes obvious that $\sigma(W)=\mathbb{R}^{+} \cup\{0\}$ and that the spectral measure of $W$ is absolutely continuous with respect to the Lebesgue measure $d a$. Furthermore, the functional calculus can be expressed by

$$
(\widehat{h(W) f})(b)=h(b) \hat{f}(b)
$$

implying that the spectral measure satisfies the relation

$$
\left.\widehat{\left(d E_{a} f\right.}\right)(b)=\delta(b-a) \hat{f}(b) d a
$$

As a consequence, the integrand in (3.8) can be written as

$$
\left\langle f, d E_{a} g\right\rangle_{L^{2}\left(\mathbb{R}^{+}, z d z\right)}=\overline{\hat{f}(a)} \hat{g}(a) \rho(a) d a
$$

with a nonnegative measurable function $\rho$. 
In order to determine $\rho$, we compute the left side of (3.10) with the help of (3.9) to obtain

$$
\begin{aligned}
& \overline{\hat{f}(a)} \hat{g}(a) \rho(a)=\int_{0}^{\infty} z d z \int_{M} \frac{d^{4} k}{(2 \pi)^{4}} \mathrm{e}^{-\mathrm{i} \omega \sqrt{z}} \overline{\hat{f}\left(k^{2}\right)} \Theta\left(k^{2}\right) \epsilon\left(k^{0}\right) \\
& \times \int_{M} \frac{d^{4} k^{\prime}}{(2 \pi)^{4}} \mathrm{e}^{\mathrm{i} \omega^{\prime} \sqrt{z}} \hat{g}\left(k^{\prime 2}\right) \delta\left(k^{\prime 2}-a\right) \epsilon\left(k^{\prime 0}\right),
\end{aligned}
$$

where $\omega$ and $\omega^{\prime}$ denote the zero components of $k$ and $k^{\prime}$, respectively. Using the scaling $a \rightarrow \lambda^{2} a, k \rightarrow \lambda k, k^{\prime} \rightarrow \lambda k^{\prime}$ and $z \rightarrow z / \lambda^{2}$, we find that

$$
\overline{\hat{f}\left(\lambda^{2} a\right)} \hat{g}\left(\lambda^{2} a\right) \rho\left(\lambda^{2} a\right)=\overline{\hat{f}\left(\lambda^{2} a\right)} \hat{g}\left(\lambda^{2} a\right) \lambda^{2} \rho(a)
$$

and thus $\rho(a)=c a$ with a positive constant $c$. Using this fact in (3.10), the abstract formula (3.8) becomes

$$
\int_{0}^{\infty} \overline{f(z)} g(z) z d z=c \int_{0}^{\infty} \overline{\hat{f}(a)} \hat{g}(a) a d a .
$$

We finally determine $c$ using the symmetry between position and momentum space together with the fact that the definitions of the Fourier transformation and its inverse differ by factors of $(2 \pi)^{4}$ (compare (2.2) and (3.3)).

We now extend the last theorem to include Dirac matrices. We thus consider similar to (3.1) a function $F$ (and analogously $G$ ) of the form

$$
F(\xi)=\frac{\$}{2} f\left(\xi^{2}\right) \Theta\left(\xi^{2}\right) \epsilon\left(\xi^{0}\right) .
$$

In the inner product we combine the two resulting factors $\$$ to a factor $z$,

$$
\langle F, G\rangle:=\int_{0}^{\infty} z \overline{f(z)} g(z) z d z
$$

Then the Fourier transform $\hat{F}(k)$, again defined by (3.3), can be written as

$$
\hat{F}(k)=\frac{\not k}{2} \hat{f}\left(k^{2}\right) \Theta\left(k^{2}\right) \epsilon\left(k^{0}\right) .
$$

The inner product in momentum space is defined by

$$
\langle\hat{F}, \hat{G}\rangle=\frac{1}{(2 \pi)^{4}} \int_{0}^{\infty} a \overline{\hat{f}(a)} \hat{g}(a) a d a .
$$


With a slight abuse of notation, we denote the Hilbert spaces corresponding to the inner products (3.12) and (3.14) again by $L^{2}(M, z d z)$ and $L^{2}(\hat{M}, a d a)$, respectively.

Corollary 3.1. (Lorentz invariant Plancherel formula, vector case). For functions of the form (3.11), the Fourier transform (3.3) is a unitary mapping from $L^{2}(M, z d z)$ to $L^{2}(\hat{M}, a d a)$. In particular, for all $F, G \in$ $L^{2}(M, z d z)$,

$$
\int_{0}^{\infty} z \overline{f(z)} g(z) z d z=\frac{1}{(2 \pi)^{4}} \int_{0}^{\infty} a \overline{\hat{f}(a)} \hat{g}(a) a d a .
$$

Proof. We can again assume that $f, g \in C_{0}^{\infty}\left(\mathbb{R}_{+}\right)$. Introducing the scalar function

$$
F_{s}(\xi)=f_{s}\left(\xi^{2}\right) \Theta\left(\xi^{2}\right) \epsilon\left(\xi^{0}\right) \text { with } \quad f_{s} \equiv f,
$$

we obviously have $F(\xi)=\$ F_{s}(\xi) / 2$. Since multiplication in position space corresponds to differentiation in momentum space, we know that

$$
\hat{F}(k)=\frac{\mathrm{i} \partial_{k}}{2} \hat{F}_{s}(k) .
$$

Representing $\hat{F}_{s}$ in the form (3.4) with an overall subscript $s$, it follows that the function $\hat{f}$ in (3.13) is given by

$$
\hat{f}(a)=2 \hat{f}_{s}^{\prime}(a) .
$$

Finally, we know that $\widehat{\xi^{2} F_{s}(\xi)}=-\square_{k} \hat{F}_{s}(k)$ and thus

$$
\widehat{z f_{s}(z)}=\hat{W} \hat{f}_{s}(a)
$$

where $\hat{W}$ is minus the wave operator in momentum space, i.e. in analogy to $(3.5)$

$$
\left(\hat{W} \hat{f}_{s}\right)(a)=-\frac{4}{a} \frac{d}{d a}\left(a^{2} \frac{d}{d a} \hat{f}_{s}\right)
$$

Using for $G$ the same notation as for $F$, we obtain

$$
\begin{aligned}
& \int_{0}^{\infty} z \overline{f(z)} g(z) z d z=\int_{0}^{\infty} \overline{z f_{s}(z)} g_{s}(z) z d z \\
& \quad \stackrel{(*)}{=} \frac{1}{(2 \pi)^{4}} \int_{0}^{\infty} \overline{z f_{s}(a)} \hat{g}_{s}(a) a d a=\frac{1}{(2 \pi)^{4}} \int_{0}^{\infty}\left(\hat{W} \overline{\hat{f}_{s}(a)}\right) \hat{g}_{s}(a) a d a \\
& \quad \stackrel{(+)}{=} \frac{1}{(2 \pi)^{4}} \int_{0}^{\infty} 4 \overline{\hat{f}_{s}^{\prime}(a)} \hat{g}_{s}^{\prime}(a) a^{2} d a=\frac{1}{(2 \pi)^{4}} \int_{0}^{\infty} a \overline{\hat{f}(a)} \hat{g}(a) a d a
\end{aligned}
$$


where in $\left(^{*}\right)$ we applied Theorem 3.1 , and in $(+)$ we used the explicit form of $\hat{W},(3.15)$, and integrated by parts.

\section{The variational principle in momentum space, connection to state stability}

Using the Lorentz invariant Plancherel formula of Corollary 3.1, we shall now transform our action and the corresponding EL equations to momentum space. The main difficulty is to treat the singularities of $A_{0}$ on the light cone. We here merely describe the regularization schematically; the detailed constructions involving a rather subtle regularization technique will be carried out in the subsequent Sections 5 and 6.

We want to apply Corollary 3.1 to the integral in (2.12). The simplest approach would be to regularize $A_{0}$ by multiplication with a characteristic function

$$
A_{0}^{\varepsilon}(\xi):=\Theta\left(\xi^{2}-\varepsilon\right) A_{0}(\xi)
$$

According to (2.15),

$$
A_{0}^{\varepsilon}(\xi)=\frac{\not}{2} f^{\varepsilon}\left(\xi^{2}\right) \Theta\left(\xi^{2}\right) \epsilon\left(\xi^{0}\right) \quad \text { with } \quad f^{\varepsilon}(z)=\Theta(z-\varepsilon) f(z) .
$$

Then

$$
\begin{aligned}
\int_{\varepsilon}^{\infty} \operatorname{Tr}\left(A_{0}^{2}\right) z d z & =\int_{0}^{\infty} \operatorname{Tr}\left(\left(A_{0}^{\varepsilon}\right)^{2}\right) z d z \\
& =\int_{0}^{\infty} f^{\varepsilon}(z)^{2} z^{2} d z=\frac{1}{(2 \pi)^{4}} \int_{0}^{\infty}\left|\widehat{f^{\varepsilon}}(a)\right|^{2} a^{2} d a
\end{aligned}
$$

where in the last step we applied Corollary 3.1. Here $\widehat{f^{\varepsilon}}$ is defined via the relation

$$
\int d^{4} \xi \Theta\left(\xi^{2}-\varepsilon\right) A_{0} \mathrm{e}^{-\mathrm{i} k \xi}=\frac{\not k}{2} \widehat{f^{\varepsilon}}\left(k^{2}\right) \Theta\left(k^{2}\right) \epsilon\left(k^{0}\right)
$$

The problem is that in (4.3) we take the Fourier transform of a function which is discontinuous on the hypersurface $\left\{\xi \mid \xi^{2}=\varepsilon\right\}$. As a consequence, the function $\widehat{f^{\varepsilon}}$ depends on the regularization in a complicated oscillatory way, making it difficult to analyze the $\varepsilon$-dependence of the last integral in $(4.2)$. 
To bypass this difficulty, we will use a different regularization method. First, we extend $A_{0}$ in the distributional sense across the light cone. More precisely, we introduce $\tilde{\mathcal{M}}(\xi)$ as in [5] by the requirements that

$$
\tilde{\mathcal{M}} \in \mathcal{S}^{\prime}(M) \quad \text { and } \quad \tilde{\mathcal{M}}(\xi)=2 A_{0}(\xi) \quad \forall \xi \notin L,
$$

where $\mathcal{S}^{\prime}(M)$ denotes the space of tempered distributions. Furthermore, $\tilde{\mathcal{M}}$ should respect the symmetry (2.14),

$$
\tilde{\mathcal{M}}(-\xi)=\tilde{\mathcal{M}}(\xi) .
$$

The Fourier transform of $\tilde{\mathcal{M}}$ is denoted by $\hat{\mathcal{M}} \in \mathcal{S}^{\prime}(\hat{M})$. Using again the symmetry argument after (3.3), we find that

$$
\operatorname{supp} \hat{\mathcal{M}} \subset \overline{\mathcal{C}} \quad \text { and } \quad \tilde{\mathcal{M}}(-k)=\tilde{\mathcal{M}}(k) .
$$

Next, we regularize $\tilde{\mathcal{M}}$ in a Lorentz invariant way which is simple and explicit both in position and momentum space. The regularized $\tilde{\mathcal{M}}$ is in $L^{2}(M, z d z)$, making it possible to apply Corollary 3.1. This allows us to rewrite the action (2.12) as

$$
\mathcal{S}=\frac{1}{(2 \pi)^{4}} \lim _{\varepsilon \searrow 0}\left(\int_{0}^{\infty} \frac{1}{4} \operatorname{Tr}\left(\hat{\mathcal{M}}^{\varepsilon}(k)^{2}\right) a d a+F_{\varepsilon}\left(\mathfrak{m}_{3}, \mathfrak{m}_{5}\right)\right),
$$

where $k \in \hat{M}$ is any vector with $k^{2}=a$. Here the new function $F_{\varepsilon}$ also involves the counter terms. For the first variation we then obtain

$$
\delta \mathcal{S}=\frac{1}{(2 \pi)^{4}} \lim _{\varepsilon \searrow 0}\left(\int_{0}^{\infty} \frac{1}{2} \operatorname{Tr}\left(\hat{\mathcal{M}}^{\varepsilon} \delta \hat{\mathcal{M}}^{\varepsilon}\right) a d a+\delta F_{\varepsilon}\left(\mathfrak{m}_{3}, \mathfrak{m}_{5}\right)\right) .
$$

According to $(4.4)$ and $(2.3)$, the distribution $\tilde{\mathcal{M}}(\xi) / 2$ coincides away from the light cone with the trace-free part of the product $P(\xi)$ times $P(-\xi)$. Since multiplication in position space corresponds to convolution in momentum space, one might expect that $\hat{\mathcal{M}}$ equals the convolution of $\hat{P}$ by itself. More precisely, the change of variables

$$
\begin{aligned}
P(\xi) P(-\xi) & =\int \frac{d^{4} p}{(2 \pi)^{4}} \int \frac{d^{4} q}{(2 \pi)^{4}} \hat{P}(p) \hat{P}(q) \mathrm{e}^{\mathrm{i}(p-q) \xi} \\
& =\int \frac{d^{4} k}{(2 \pi)^{4}}\left(\int \frac{d^{4} q}{(2 \pi)^{4}} \hat{P}(k+q) \hat{P}(q)\right) \mathrm{e}^{\mathrm{i}(p-q) \xi}
\end{aligned}
$$

suggests that it should be helpful to decompose $\hat{\mathcal{M}}^{\varepsilon}$ as

$$
\hat{\mathcal{M}}^{\varepsilon}(k)=\hat{\mathcal{F}}(k)+\hat{\mathcal{N}}^{\varepsilon}(k),
$$




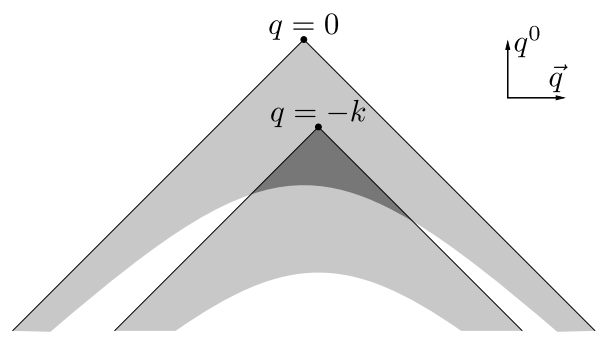

Figure 1: The convolution integral in (4.10).

where we set

$$
\hat{\mathcal{F}}(k):=2 \int \frac{d^{4} q}{(2 \pi)^{4}}(\hat{P}(k+q) \hat{P}(q))_{0}, \quad \text { defined if } k^{2}>0 .
$$

Here the subscript zero means that we take only the vector component, and the new function $\hat{\mathcal{N}}^{\varepsilon}$ involves the dependence on the regularization. As the poles of $\hat{\mathcal{M}}$ are characterized by $\mathfrak{m}_{3}$ and $\mathfrak{m}_{5}$ (see $(2.8)$ ), it is not astonishing that $\hat{\mathcal{N}}^{\varepsilon}$ will depend only on the parameters $\mathfrak{m}_{3}, \mathfrak{m}_{5}$ and $\varepsilon$. Using that $\hat{P}$ is supported on the lower mass shells (see (2.1)), one sees that the integrand in (4.10) is compactly supported (see figure 1 in the case when $k \in \mathcal{C}^{\vee}$; the case $k \in \mathcal{C}^{\wedge}$ is similar). As a consequence, the integral in (4.10) will be finite. Since $\hat{\mathcal{M}}^{\varepsilon} \rightarrow \hat{\mathcal{M}}$ as $\varepsilon \searrow 0$, it is obvious from (4.9) that $\hat{\mathcal{N}}^{\varepsilon}$ converges in this limit to a function $\hat{\mathcal{N}}(k)$ defined if $k^{2}>0$. Using (4.9) in (4.8), we obtain

$$
\begin{aligned}
\delta \mathcal{S}= & \frac{1}{(2 \pi)^{4}} \lim _{\varepsilon \searrow 0} \int_{0}^{\infty} a d a \frac{1}{2} \operatorname{Tr}\left(\hat{\mathcal{M}}^{\varepsilon}(k) \delta \hat{\mathcal{F}}(k)\right) \\
& +\frac{1}{(2 \pi)^{4}} \lim _{\varepsilon \searrow 0}\left(\int_{0}^{\infty} \frac{1}{2} \operatorname{Tr}\left(\hat{\mathcal{M}}^{\varepsilon} \delta \hat{\mathcal{N}}^{\varepsilon}\right) a d a+\delta F_{\varepsilon}\left(\mathfrak{m}_{3}, \mathfrak{m}_{5}\right)\right)
\end{aligned}
$$

We shall see that in (4.11) the regularization can be removed. In (4.12), on the other hand, the integral diverges as $\varepsilon \searrow 0$, but this divergence is compensated by the counter terms in $\delta F_{\varepsilon}$. We thus obtain the following result, where for notational convenience we omit the irrelevant prefactors $(2 \pi)^{-4}$.

Lemma 4.1. The first variation of action (2.12) can be written as

$$
\delta \mathcal{S}=\int_{0}^{\infty} a d a \int \frac{d^{4} q}{(2 \pi)^{4}} \operatorname{Tr}\left(\hat{\mathcal{M}}(k) \delta(\hat{P}(k+q) \hat{P}(q))_{0}\right)+\delta F\left(\mathfrak{m}_{3}, \mathfrak{m}_{5}\right)
$$

where $k \in \hat{M}$ is any vector with $k^{2}=a$, and $\delta F$ denotes the variation of the function $F$ in Definition 2.1. 
This concludes our sketch of the regularization procedure. This sketch will be our guideline for the constructions in Sections 5 and 6. The proof of Lemma 4.1 will be given at the end of Section 6 .

We next consider the variation of the product in (4.13). Changing variables

$$
\int d^{4} q(\delta \hat{P}(k+q)) \hat{P}(q)=\int d^{4} q(\delta \hat{P}(q)) \hat{P}(-k+q)
$$

and using that commuting the two factors in the integrand does not change their vector component, we find that varying the first factor is the same as varying the second factor if we flip the sign of $k$. In view of (4.6) we can thus write the variation of the action as

$$
\begin{aligned}
\delta \mathcal{S}= & \int_{0}^{\infty} a d a \int \frac{d^{4} q}{(2 \pi)^{4}} \operatorname{Tr}([\hat{\mathcal{M}}(k) \hat{P}(k+q) \\
& +\hat{\mathcal{M}}(-k) \hat{P}(-k+q)] \delta \hat{P}(q)) \\
& +\delta F\left(\mathfrak{m}_{3}, \mathfrak{m}_{5}\right)
\end{aligned}
$$

where $k \in \hat{M}$ is an arbitrary vector with $k^{2}=a$. Note that we here omitted the subscript zero. This does not change the value of the trace, because the factor $\hat{\mathcal{M}}$ only has a vector component.

The following geometric argument is crucial for getting an understanding of the EL equations. It suffices to consider the first summand in the square bracket in (4.14), because the second term is then obtained by flipping the sign of $k$. Using the support property in (4.6), we may insert the integral over a $\delta$-distribution,

$$
\begin{aligned}
& \int_{0}^{\infty} a d a \int d^{4} q \hat{\mathcal{M}}(k) \hat{P}(k+q) \delta \hat{P}(q) \\
& \quad=\int_{0}^{\infty} a d a \int_{0}^{\infty} d b \int d^{4} q \delta\left(q^{2}-b\right) \hat{\mathcal{M}}(k) \hat{P}(k+q) \delta \hat{P}(q) .
\end{aligned}
$$

For fixed $a, b$ and $k \in \mathbb{C}$, the $q$-integral extends over a compact subset of the hyperbola $\left\{q \mid q^{2}=b\right\}$ (see figure 2). Any two vectors on this hyperbola can be transformed into each other by a Lorentz boost. Thus, using Lorentz symmetry, we can assume that the vector $q$ is fixed. Instead, the vector $k$ will no longer be constant, but it will run over a compact subset of the hyperbola $\left\{k \mid k^{2}=a\right\}$ (see figure 3). Taking into account the relative sizes of the $q$-subset and the corresponding $k$-subset, we obtain the transformation 

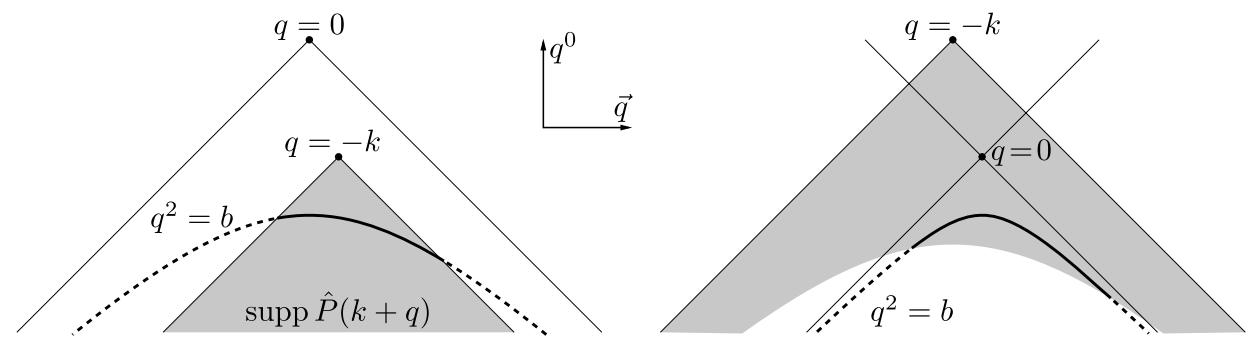

Figure 2: The $q$-integral in (4.15) in the cases $k \in \mathcal{C}^{\vee}$ (left) and $k \in \mathcal{C}^{\wedge}$ (right).
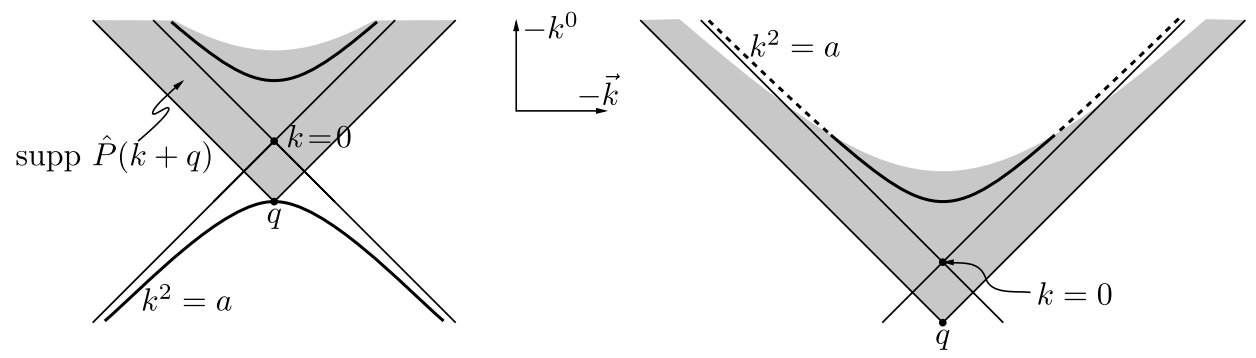

Figure 3: The corresponding $k$-integrals in the two cases of figure 2 .

rule

$$
\int_{0}^{\infty} a d a \int d^{4} q \cdots=\int_{0}^{\infty} b d b \int_{\mathcal{C}^{\wedge} \text { or } \mathcal{C}^{\vee}} d^{4} k \cdots,
$$

where on the right we choose any vector $q \in \mathcal{C}^{\wedge}$ with $q^{2}=b$, and the $k$-integral goes over $\mathcal{C}^{\vee}$ or $\mathcal{C}^{\wedge}$, depending on whether we started with a vector $k$ on the lower or upper mass cone. Combining the contributions of the two summands in the square bracket in (4.14), we obtain a $k$-integral over $\mathcal{C}$. Finally, the support property in (4.6) allows us to extend the $k$-integration to all of $\hat{M}$. We thus obtain

$$
\delta \mathcal{S}=\int_{0}^{\infty} b d b \int \frac{d^{4} k}{(2 \pi)^{4}} \operatorname{Tr}(\hat{\mathcal{M}}(k) \hat{P}(k+q) \delta \hat{P}(q))+\delta F\left(\mathfrak{m}_{3}, \mathfrak{m}_{5}\right)
$$

Next, it is convenient to transform the $k$-integral to a convolution integral

$$
\int \frac{d^{4} k}{(2 \pi)^{4}} \hat{\mathcal{M}}(k) \hat{P}(k+q)=\int \frac{d^{4} k}{(2 \pi)^{4}} \hat{\mathcal{M}}(k) \hat{P}(q-k)=: \quad(\hat{\mathcal{M}} * \hat{P})(q)
$$


where we flipped the sign of $k$ and used the symmetry property of $\hat{\mathcal{M}}$ in (4.6). Then we can write the variation of the action in the more compact form

$$
\delta \mathcal{S}=\int_{0}^{\infty} \operatorname{Tr}((\hat{\mathcal{M}} * \hat{P})(q) \delta \hat{P}(q)) b d b+\delta F\left(\mathfrak{m}_{3}, \mathfrak{m}_{5}\right)
$$

Our last step is to treat the variation of the function $F$. According to the chain rule

$$
\delta F\left(\mathfrak{m}_{3}, \mathfrak{m}_{5}\right)=D_{1} F\left(\mathfrak{m}_{3}, \mathfrak{m}_{5}\right) \delta \mathfrak{m}_{3}+D_{2} F\left(\mathfrak{m}_{3}, \mathfrak{m}_{5}\right) \delta \mathfrak{m}_{5}
$$

It is an important observation that relations (4.4) determine $\tilde{\mathcal{M}}$ only up to singular contributions on the light cone. More specifically, there is the natural freedom to modify $\tilde{\mathcal{M}}(\xi)$ by $\delta^{\prime}$ - and $\delta$-contributions of the form

$$
\tilde{\mathcal{M}}(\xi) \asymp c_{0} \Varangle \delta^{\prime}\left(\xi^{2}\right) \epsilon\left(\xi^{0}\right)+c_{1} \Varangle \delta\left(\xi^{2}\right) \epsilon\left(\xi^{0}\right)
$$

As we shall see later (see Lemma 5.6), by modifying $c_{0}$ and $c_{1}$ appropriately, we can precisely absorb the term (4.17) which arises in the variation of $F$. Conversely, the freedom to choose the function $F$ in $(2.12)$ can be regarded as taking into account the fact that the distribution $\tilde{\mathcal{M}}$ is determined only modulo the contribution (4.18). In what follows, we shall always omit the term $\delta F\left(\mathfrak{m}_{3}, \mathfrak{m}_{5}\right)$ in (4.16), giving the following result.

Proposition 4.1. The first variation of the action $\mathcal{S},(2.12)$, can be written in momentum space as

$$
\delta \mathcal{S}=2 \int_{0}^{\infty} \operatorname{Tr}(\hat{Q}(q) \delta \hat{P}(q)) b d b
$$

where $q \in \mathcal{C}^{\wedge}$ is any vector in the lower mass cone with $q^{2}=b$ and

$$
\hat{Q}(q)=\frac{1}{2}(\hat{\mathcal{M}} * \hat{P})(q):=\frac{1}{2} \int \frac{d^{4} p}{(2 \pi)^{4}} \hat{\mathcal{M}}(p) \hat{P}(q-p) .
$$

Here $\hat{\mathcal{M}}$ is the Fourier transform of the distribution $\tilde{\mathcal{M}}$ as introduced by (4.4), for specific values of the free parameters $c_{0}$ and $c_{1}$ in (4.18).

To fully understand (4.19) and (4.20), one should observe that, according to $(2.1)$, the factor $\delta \hat{P}(q)$ in (4.19) is supported in $\mathcal{C}^{\wedge}$. But for $q$ in the lower mass cone, the convolution integral in (4.20) is finite, because the integrand 


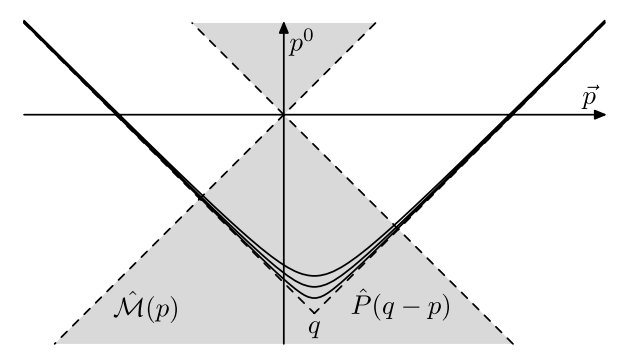

Figure 4: The convolution $\hat{\mathcal{M}} * \hat{P}$.

is compactly supported (see figure 4). The Fourier transform of $\hat{Q}$ is simply the product

$$
Q(\xi)=\frac{1}{2} \tilde{\mathcal{M}}(\xi) P(\xi) .
$$

The distribution $Q(y-x)$ is precisely the kernel of the operator $Q$ which appears in the EL equations $[P, Q]=0$ corresponding to the variational principle introduced in $[1, \S 3.5]$.

The notion of state stability imposes conditions on the eigenvalues of the matrix $\hat{Q}(q)$. For the sake of self-consistency we here restate the definition as given in [1, Definition. 5.6.2].

Definition 4.1. The fermionic projector of the vacuum is called state stable if the corresponding operator $\hat{Q}(k)$ is well-defined in the lower mass cone $\mathcal{C}^{\wedge}$ and can be written as

$$
\hat{Q}(k)=a \frac{\not k}{|k|}+b
$$

(where $|k| \equiv \sqrt{k^{2}}$ ) with continuous real functions $a$ and $b$ on $\mathcal{C}^{\wedge}$ having the following properties:

(i) $a$ and $b$ are Lorentz invariant,

$$
a=a\left(k^{2}\right), \quad b=b\left(k^{2}\right) .
$$

(ii) $a$ is nonnegative.

(iii) The function $a+b$ is minimal on the mass shells,

$$
(a+b)\left(m_{\alpha}^{2}\right)=\inf _{q \in \mathcal{C}^{\wedge}}(a+b)\left(q^{2}\right) \quad \forall \alpha \in\{1, \ldots, g\} .
$$

In our setting, representation (4.22) as well as (i) are obvious, and thus we need to analyze (ii) and (iii). The next theorem shows that the EL equations 
corresponding to the Lorentz invariant variational principle of Definition 2.2 give a necessary condition for state stability. Our proof will also explain the detailed form of constraint (2.13).

Theorem 4.1. The fermionic projector is a critical point of the variational principle of Definition 2.2 if and only if the functions $a$ and $b$ in representation (4.22) have the following properties:

(a) $(a+b)\left(m_{\alpha}^{2}\right)=(a+b)\left(m_{\beta}^{2}\right) \quad \forall \alpha, \beta \in\{1, \ldots, g\}$,

(b) $(a+b)^{\prime}\left(m_{\alpha}^{2}\right)=0 \quad \forall \alpha \in\{1, \ldots, g\}$.

Proof. Using ansatz (2.1), we find

$$
\frac{1}{2} \int_{0}^{\infty} \operatorname{Tr}(\hat{Q}(q) \hat{P}(q)) b d b=\frac{1}{2} \sum_{\beta=1}^{g} \rho_{\beta} m_{\beta}^{3} \operatorname{Tr}\left(\hat{Q}\left(q_{\beta}\right) \frac{q_{\beta}+m_{\beta}}{m_{\beta}}\right),
$$

where $q, q_{\beta} \in \mathcal{C}^{\wedge}$ are any vectors with $q^{2}=b$ and $q_{\beta}^{2}=m_{\beta}^{2}$. Using (4.22), this simplifies to

$$
\frac{1}{2} \int_{0}^{\infty} \operatorname{Tr}(\hat{Q}(q) \hat{P}(q)) b d b=2 \sum_{\beta=1}^{g} \rho_{\beta} m_{\beta}^{3}(a+b)\left(m_{\beta}^{2}\right)
$$

According to Proposition 4.1, $\delta \mathcal{S}$ is obtained from (4.24) by varying $\rho_{\beta}$ and $m_{\beta}$, keeping the operator $\hat{Q}$ fixed. Equivalently, we can vary (4.25) for fixed functions $a$ and $b$. We treat constraint (2.13) as in (2.17) with a Lagrange multiplier $\lambda$. Varying the weights $\rho_{\beta}$ gives the conditions

$$
2 m_{\beta}^{3}(a+b)\left(m_{\beta}^{2}\right)=\lambda m_{\beta}^{3} \quad \forall \beta \in\{1, \ldots, g\},
$$

proving (a). Similarly, varying the masses $m_{\beta}$ and using (4.26) gives (b).

Properties (a) and (b) in this theorem are weaker than condition (iii) in the definition of state stability, because (4.23) implies in addition that the points $m_{\beta}^{2}$ are absolute minima of the function $a+b$. Moreover, our theorem makes no statement on condition (ii). Nevertheless, the above theorem shows that our Lorentz invariant variational principle is closely related to state stability, and it seems a promising strategy for satisfying state stability to construct minimizers of our variational principle, and then to select those minimizers which satisfy the additional conditions of Definition 4.1.

In order to get stronger conditions from our variational principle, one needs to consider more general variations of the fermionic projector. A convenient method is to consider a "test Dirac sea" of infinitesimal weight and 
of possibly negative mass. Thus we consider instead of (2.1) the fermionic projector

$$
\hat{P}(k)=\sum_{\beta=1}^{g+1} \rho_{\beta}\left(\not k+m_{\beta}\right) \delta\left(k^{2}-m_{\beta}^{2}\right) \Theta\left(-k^{0}\right)
$$

and assume that $\rho_{g+1}$ vanishes for our unperturbed fermionic projector. We vary $\rho_{g+1}$ for given $m_{g+1} \in \mathbb{R}$ and introduce the function $V$ by

$$
V\left(m_{g+1}\right)=\left.\frac{1}{2 m_{g+1}^{3}} \frac{\partial}{\partial \rho_{g+1}} \mathcal{S}\right|_{\rho_{g+1}=0} .
$$

We refer to $V$ as the variation density. We point out that the $(g+1)^{\text {st }}$ Dirac sea should not be considered as being of physical significance; it is merely a mathematical tool to generate variations of the action.

Theorem 4.2. The functions $a$ and $b$ in (4.22) are related to the variation density by

$$
V(m)=\epsilon(m) a\left(m^{2}\right)+b\left(m^{2}\right)
$$

Proof. Taking into account that $m_{g+1}$ may be negative, (4.25) is modified to

$$
\frac{1}{2} \int_{0}^{\infty} \operatorname{Tr}(\hat{Q}(q) \hat{P}(q)) b d b=2 \sum_{\beta=1}^{g} \rho_{\beta} m_{\beta}^{3}\left(\epsilon\left(m_{\beta}\right) a\left(m_{\beta}^{2}\right)+b\left(m_{\beta}^{2}\right)\right)
$$

Differentiating with respect to $\rho_{g}$ gives the result.

In view of this theorem, conditions (ii) and (iii) are can be expressed in terms of the variation density by
(ii') $V(m) \geq V(-m)$
$\forall m \in \mathbb{R}^{+}$,
(iii') $V\left(m_{\beta}\right) \leq \inf _{\mathbb{R}^{+}} V$
$\forall \beta \in\{1, \ldots g\}$.

\section{Convolutions of Lorentz invariant distributions}

We saw in the previous section that it is useful to rewrite products in position space as convolutions in momentum space

$$
\widehat{F G}(q)=(\hat{F} * \hat{G}):=\int \frac{d^{4} k}{(2 \pi)^{4}} \hat{F}(k) \hat{G}(q-k)
$$


and to analyze the resulting convolution integrals (see (4.9) and (4.20)). In this section we study such convolution integrals systematically. The distributions of interest like (3.4) or (3.13) are Lorentz invariant and are supported in the mass cone $\mathcal{C}$. It is helpful to decompose these distributions into the sum of a distribution supported in the upper mass cone and a distribution supported in the lower mass cone. This gives rise to the following definition.

Definition 5.1. A Lorentz-invariant distribution $\hat{F} \in \mathcal{S}^{\prime}(\hat{M})$ is called negative if it is supported in $\mathcal{C}^{\wedge}$ and can be written as

$$
\hat{F}(k)=f\left(k^{2}\right) \Theta\left(k^{2}\right) \Theta\left(-k^{0}\right)
$$

with a real-valued distribution $f \in \mathcal{S}^{\prime}\left(\mathbb{R}^{+}\right)$. Similarly, $\hat{F}(k)$ is called positive if $\hat{F}(-k)$ is negative.

We remark for clarity that representation (5.2) poses an extra condition which in particular excludes all distributions supported at the origin. For example, the distribution $\delta^{4}(k)$ is Lorentz invariant, supported in $\mathcal{C}^{\wedge}$, but it cannot be represented in the form (5.2).

Since $\hat{F}$ is completely characterized by $f$, it is most convenient to work only with the small letters. Note that for notational convenience, the function $f$ does not carry a hat; to clarify momentum space we shall write out the arguments $a:=k^{2}, \ldots$. In our computations we will use a compact notation which reminds of the corresponding operations in position space. The following list shows what operations on $f$ and $g$ mean for the corresponding distributions $\hat{F}$ and $\hat{G}$ :

$$
\left.\begin{array}{ccc}
(f \cdot g)(a) & \Longleftrightarrow & (F * G)(k), \\
\bar{f}(a) & \Longleftrightarrow & F(-k), \\
\not \partial f(a) & \Longleftrightarrow & i \not k F(k) .
\end{array}\right\}
$$

The notation $\bar{f}$ cannot lead to confusion because $f(a)$ is real valued, and therefore the only reasonable meaning is to take the complex conjugate in position space, which corresponds to flipping the sign of $k$. If $f$ is negative, then clearly $\bar{f}$ is positive. Keeping this in mind, we shall always assume that $f$ and $g$ correspond to negative distributions, whereas positive distributions are denoted by $\bar{f}$ and $\bar{g}$. The operator $\not \partial$ is convenient for handling Dirac matrices.

If $\hat{F}$ and $\hat{G}$ are both negative (or both positive), the integrand of the convolution integral (5.1) is compact (see figure 5 (left)). If on the other hand one factor is positive and the other is negative, the support of the 

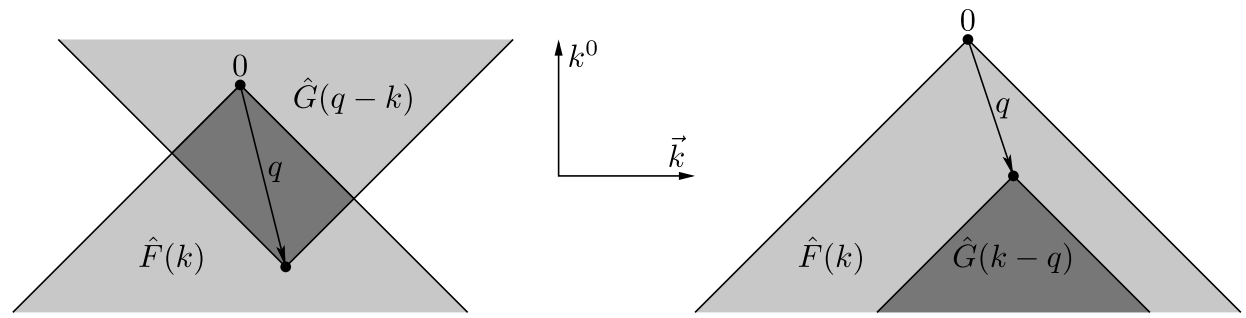

Figure 5: Convolution of two negative distributions (left) and a mixed convolution (right).

integrand will in general be non-compact (see figure 5 (right)). These two cases are essentially different and will be treated separately in Sections 5.1 and 5.2. In Section 5.3 we shall analyze the distribution $\hat{\mathcal{M}}$, which already appeared in (4.4) and (4.5).

\subsection{Convolutions of negative distributions}

We introduce the function $\Delta(a, b, c)$ by

$$
\Delta=a^{2}+b^{2}+c^{2}-2(a b+a c+b c) .
$$

Note that $\Delta$ is symmetric in its three arguments.

Lemma 5.1. Suppose that $f$ and $g$ are negative distributions. Then the following convolutions are also negative and are given explicitly by

$$
\begin{aligned}
(f \cdot g)(a) & =\frac{1}{32 \pi^{3}} \int_{0}^{a} d c f(c) \int_{0}^{(\sqrt{a}-\sqrt{c})^{2}} d b g(b) \frac{\sqrt{\Delta}}{a}, \\
(\not \partial f \cdot g)(a) & =(\not \partial \alpha)(a), \\
\alpha(a) & =\frac{1}{32 \pi^{3}} \int_{0}^{a} d c f(c) \int_{0}^{(\sqrt{a}-\sqrt{c})^{2}} d b g(b) \sqrt{\Delta} \frac{a-b+c}{2 a^{2}}, \\
(f \cdot \not \partial g)(a) & =(\not \partial)(a), \\
\beta(a) & =\frac{1}{32 \pi^{3}} \int_{0}^{a} d c f(c) \int_{0}^{(\sqrt{a}-\sqrt{c})^{2}} d b g(b) \sqrt{\Delta} \frac{a+b-c}{2 a^{2}}, \\
\left(\partial_{k} f \cdot \partial^{k} g\right)(a) & =\frac{1}{32 \pi^{3}} \int_{0}^{a} d c f(c) \int_{0}^{(\sqrt{a}-\sqrt{c})^{2}} d b g(b) \sqrt{\Delta} \frac{c+b-a}{2 a} .
\end{aligned}
$$

Proof. Clearly, the convolutions are again Lorentz invariant, and a geometric argument (see figure 5 (left)) immediately yields that they are again 
negative. To calculate the convolutions, we choose any $q \in \mathcal{C}^{\wedge}$ with $q^{2}=a$. Then

$$
\begin{aligned}
(f \cdot g)(a)= & \int \frac{d^{4} k}{(2 \pi)^{4}} \hat{F}(k) \hat{G}(q-k) \\
= & \int_{0}^{\infty} d c \int_{0}^{\infty} d b f(c) g(b) \int \frac{d^{4} k}{(2 \pi)^{4}} \delta\left(k^{2}-c\right) \Theta\left(-k^{0}\right) \\
& \times \delta\left((q-k)^{2}-b\right) \Theta\left(k^{0}-q^{0}\right) \\
= & \int_{0}^{\infty} d c \int_{0}^{\infty} d b f(c) g(b) \int \frac{d^{4} k}{(2 \pi)^{4}} \delta\left(k^{2}-c\right) \Theta\left(-k^{0}\right) \\
& \times \delta\left(q^{2}-2 q k+c-b\right) \Theta\left(k^{0}-q^{0}\right) .
\end{aligned}
$$

Due to Lorentz symmetry, we can assume that $q$ points in the direction of $k^{0}$. Introducing polar coordinates $\left(\omega=k^{0}, p=|\vec{k}|, \Omega \in S^{2}\right)$, we can carry out the momentum integrals

$$
\begin{aligned}
(f \cdot g)(a)= & \frac{1}{4 \pi^{3}} \int_{0}^{\infty} d c \int_{0}^{\infty} d b f(c) g(b) \\
& \times \int_{q_{0}}^{0} d \omega \int_{0}^{\infty} p^{2} d p \delta\left(\omega^{2}-p^{2}-c\right) \delta(a+2 \omega \sqrt{a}+c-b) \\
= & \frac{1}{4 \pi^{3}} \int_{0}^{\infty} d c \int_{0}^{\infty} d b f(c) g(b) \Theta\left(\omega_{0}^{2}-c\right) \chi_{[-\sqrt{a}, 0]}\left(\omega_{0}\right) \frac{p_{0}}{4 \sqrt{a}}
\end{aligned}
$$

where $\chi$ is the characteristic function, and $\omega_{0}$ and $p_{0}$ are determined by the conditions

$$
\omega_{0}=\frac{c+a-b}{-2 \sqrt{a}}, \quad p_{0}^{2}=\omega_{0}^{2}-c .
$$

A short calculation shows that $p_{0}^{2}=\Delta /(4 a)$ with $\Delta$ according to (5.4). Using that the Heaviside and the characteristic function in (5.9) vanish unless $\sqrt{a} \geq \sqrt{c}+\sqrt{b}$, we obtain $(5.5)$.

In order to derive (5.6), we first compute

$$
\begin{aligned}
(\not \partial f \cdot g)(a)= & \int \frac{d^{4} k}{(2 \pi)^{4}} \mathrm{i} \not k \hat{F}(k) \hat{G}(q-k) \\
= & \int_{0}^{\infty} d c \int_{0}^{\infty} d b f(c) g(b) \int \frac{d^{4} k}{(2 \pi)^{4}} \mathrm{i} \not k \\
& \times \delta\left(k^{2}-c\right) \delta\left(q^{2}-2 q k+c-b\right) .
\end{aligned}
$$


We again choose a reference frame with $q=(-\sqrt{a}, \overrightarrow{0})$ and introduce polar coordinates $\left(\omega=k^{0}, p=|\vec{k}|, \Omega \in S^{2}\right)$. Then

$$
(\not \partial f \cdot g)(a)=\frac{1}{4 \pi^{3}} \int_{0}^{\infty} d c \int_{0}^{\infty} d b f(c) g(b) \mathrm{i} \omega_{0} \gamma^{0} \Theta\left(\omega_{0}^{2}-c\right) \mathbb{1}_{[-\sqrt{a}, 0]}\left(\omega_{0}\right) \frac{p_{0}}{4 \sqrt{a}}
$$

with $\omega_{0}$ and $p_{0}$ according to (5.10). Note that, due to spherical symmetry, the contribution $\sim \vec{\gamma}$ vanishes. A straightforward computation yields

$$
\begin{aligned}
(\not \partial f \cdot g)(a)= & \frac{1}{32 \pi^{3}} \int_{0}^{a} d c \int_{0}^{(\sqrt{a}-\sqrt{c})^{2}} d b f(c) g(b)\left(-\mathrm{i} \sqrt{a} \gamma^{0}\right) \frac{a-b+c}{2 a} \\
& \times \frac{\sqrt{\Delta}}{a}=(\not \partial \alpha)(a)
\end{aligned}
$$

with $\alpha$ as in (5.6). Relation (5.7) immediately follows from (5.6) using the symmetry in $f$ and $g$.

To derive (5.8), we again assume that $q$ points into the direction of $k^{0}$. Then, exploiting the spherical symmetry,

$$
\begin{aligned}
\left(\partial_{k} f \cdot \partial^{k} g\right)(a)= & \int \frac{d^{4} k}{(2 \pi)^{4}} \mathrm{i} k_{l} \hat{F}(k) \mathrm{i}\left(q^{l}-k^{l}\right) \hat{G}(q-k) \\
= & \int_{0}^{\infty} d c \int_{0}^{\infty} d b f(c) g(b) \int \frac{d^{4} k}{(2 \pi)^{4}}(-k q+c) \\
& \times \delta\left(k^{2}-a\right) \delta\left(q^{2}-2 q k+c-b\right) \\
= & \frac{1}{4 \pi^{3}} \int_{0}^{\infty} d c \int_{0}^{\infty} d b f(c) g(b)\left(\omega_{0} \sqrt{a}+c\right) \Theta\left(\omega_{0}^{2}-c\right) \\
& \times \chi_{[-\sqrt{a}, 0]}\left(\omega_{0}\right) \frac{p_{0}}{4 \sqrt{a}},
\end{aligned}
$$

and a short computation gives (5.8).

\subsection{Mixed convolutions}

We shall now analyze the convolution $f \cdot \bar{g}$ of a positive and a negative distribution. We begin with the case $q \in \mathcal{C}^{\wedge}$ where the momentum is in the lower mass cone. This case is unproblematic because the integrand of the convolution integral (5.1) becomes compact if we assume that $f\left(k^{2}\right)$ vanishes identically for sufficiently large $k^{2}$. Making this additional assumption, we can use the same method as in Lemma 5.1. 
Lemma 5.2. Suppose that $f$ and $g$ are negative distributions and that $f\left(k^{2}\right)$ vanishes identically for large $k^{2}$. Then for $q \in \mathcal{C}^{\wedge}$ and setting $a=q^{2} \geq 0$, the following convolutions are well-defined and given explicitly by

$$
\begin{aligned}
(f \cdot \bar{g})(a) & =\frac{1}{32 \pi^{3}} \int_{a}^{\infty} d c f(c) \int_{0}^{(\sqrt{c}-\sqrt{a})^{2}} d b g(b) \frac{\sqrt{\Delta}}{a} \\
(\not \partial f \cdot \bar{g})(a) & =(\not \partial \alpha)(a), \\
\alpha(a) & =\frac{1}{32 \pi^{3}} \int_{a}^{\infty} d c f(c) \int_{0}^{(\sqrt{c}-\sqrt{a})^{2}} d b g(b) \sqrt{\Delta} \frac{a-b+c}{2 a^{2}}, \\
(f \cdot \not \partial \bar{g})(a) & =(\not \partial \beta)(a), \\
\beta(a) & =\frac{1}{32 \pi^{3}} \int_{a}^{\infty} d c f(c) \int_{0}^{(\sqrt{c}-\sqrt{a})^{2}} d b g(b) \sqrt{\Delta} \frac{a+b-c}{2 a^{2}} \\
\left(\partial_{k} f \cdot \partial^{k} \bar{g}\right)(a) & =\frac{1}{32 \pi^{3}} \int_{a}^{\infty} d c f(c) \int_{0}^{(\sqrt{c}-\sqrt{a})^{2}} d b g(b) \sqrt{\Delta} \frac{c+b-a}{2 a} .
\end{aligned}
$$

Proof. The momentum vector $q \in \mathcal{C}^{\wedge}$ satisfies the inequality $q^{0}<0$ and thus

$$
\begin{aligned}
(f \cdot \bar{g})(a)= & \int \frac{d^{4} k}{(2 \pi)^{4}} \hat{F}(k) \hat{G}(k-q) \\
= & \int_{0}^{\infty} d c \int_{0}^{\infty} d b f(c) g(b) \int \frac{d^{4} k}{(2 \pi)^{4}} \delta\left(k^{2}-c\right) \Theta\left(-k^{0}\right) \\
& \times \delta\left((k-q)^{2}-b\right) \Theta\left(q^{0}-k^{0}\right) \\
= & \int_{0}^{\infty} d c \int_{0}^{\infty} d b f(c) g(b) \int \frac{d^{4} k}{(2 \pi)^{4}} \delta\left(k^{2}-c\right) \\
& \times \delta\left(c-2 q k+q^{2}-b\right) \Theta\left(q^{0}-k^{0}\right) .
\end{aligned}
$$

Due to Lorentz invariance, we can assume that $q=(-\sqrt{a}, \overrightarrow{0})$. Again choosing polar coordinates $(\omega, p, \Omega)$, we obtain

$$
\begin{aligned}
(f \cdot \bar{g})(a)= & \frac{1}{4 \pi^{3}} \int_{0}^{\infty} d c \int_{0}^{\infty} d b f(c) g(b) \\
& \times \int_{-\infty}^{-\sqrt{a}} d \omega \int_{0}^{\infty} p^{2} d p \delta\left(\omega^{2}-p^{2}-c\right) \delta(c+2 \omega \sqrt{a}+a-b)
\end{aligned}
$$




$$
\begin{aligned}
= & \frac{1}{8 \pi^{3}} \int_{0}^{\infty} d c \int_{0}^{\infty} d b f(c) g(b) \\
& \times \int_{-\infty}^{-\sqrt{a}} d \omega \Theta\left(\omega^{2}-c\right) \sqrt{\omega^{2}-c} \delta\left(\omega^{2}-p^{2}-c\right) \\
& \times \delta(c+2 \omega \sqrt{a}+a-b) \\
= & \frac{1}{8 \pi^{3}} \int_{0}^{\infty} d c \int_{0}^{\infty} d b f(c) g(b) \sqrt{\omega_{0}^{2}-c} \frac{1}{2 \sqrt{a}} \\
& \times \Theta\left(\omega_{0}^{2}-c\right) \Theta(c-b-a),
\end{aligned}
$$

where we set

$$
\omega_{0}=\frac{a-b+c}{-2 \sqrt{a}} .
$$

A straightforward calculation gives (5.11).

Relations (5.12)-(5.14) are obtained with the same method as the corresponding relations (5.6)-(5.8) in Lemma 5.1.

This lemma can also be applied in the case when $q$ in the upper mass cone $\mathcal{C}^{\vee}$. Namely, changing the variables $k-q \rightarrow q$ in (5.15), one sees that flipping the sign of $q$ corresponds to exchanging $f$ and $g$. Hence the corresponding formulas are obtained if in (5.11) and (5.14) we exchange $f$ and $g$ on the right.

We come to the most interesting case that $q$ lies outside the mass cone, $q^{2}<0$. Since the hyperbolas $\left\{k^{2}=c, k^{0}<0\right\}$ and $\left\{(k-q)^{2}=c^{\prime},\left(k^{0}-\right.\right.$ $\left.\left.q^{0}\right)<0\right\}$ (for $c, c^{\prime}>0$ ) have a non-compact intersection, in this case the convolution integral (5.1) necessarily diverges for any nontrivial distributions $\hat{F}$ and $\hat{G}$. Our method to bypass this problem is to first break the Lorentz invariance by a regularization. This makes the convolution integral (5.1) finite. Then we will subtract suitable counter terms which are supported on the light cone. After that, we can remove the regularization. We thus obtain Lorentz invariant formulas for the convolution integrals, which have to be understood "modulo infinite contributions supported on the light cone." This method of disregarding contributions on the light cone is motivated from the fact that we computed the pointwise product in (2.3) only away from the light cone, with the intention of then extending the resulting function in the distributional sense across the light cone (4.4). The resulting distributions depend only on (2.3) away from the light cone. Therefore, it is irrelevant to us how the regularized quantities behave on the light cone as the regularization is removed. This is why we can simply subtract divergent terms that are supported on the light cone. 
To regularize $\hat{F}$, we set

$$
\left(f^{\varepsilon}\right)(k)=f\left(k^{2}\right) \mathrm{e}^{\varepsilon k^{0}}
$$

and introduce the distribution $\hat{F} \in \mathcal{S}^{\prime}(\hat{M})$ in analogy to $(5.2)$ by

$$
\hat{F}^{\varepsilon}(k)=f^{\varepsilon}(k) \Theta\left(k^{2}\right) \Theta\left(-k^{0}\right)
$$

In this way, we can use the previous notation, with the only difference that the functions $f^{\varepsilon}$ and $g^{\varepsilon}$ are no longer Lorentz invariant and are thus no longer functions of $q^{2}$, but of $q \in \hat{M}$. For simplicity, we assume that $f$ and $g$ have compact support.

Lemma 5.3. Suppose that $f\left(k^{2}\right)$ and $g\left(k^{2}\right)$ are negative distributions which vanish identically for large $k^{2}$. Then for $q \in \hat{M} \backslash \mathcal{C}$ and setting $a=q^{2} \leq 0$, the following formulas hold for the products of the corresponding regularized distributions (5.16) and (5.17):

$$
\begin{aligned}
\left(f^{\varepsilon} \cdot \overline{g^{\varepsilon}}\right)(q) & =\frac{1}{32 \pi^{3}} \int_{0}^{\infty} d c f(c) \int_{0}^{\infty} d b g(b) H_{\varepsilon}(q, b, c) \\
\left(\partial_{k} f^{\varepsilon} \cdot \partial^{k} \overline{g^{\varepsilon}}\right)(q) & =\frac{1}{32 \pi^{3}} \int_{0}^{\infty} d c f(c) \int_{0}^{\infty} d b g(b) \frac{b+c-a}{2} H_{\varepsilon}(q, b, c),
\end{aligned}
$$

where the function $H_{\varepsilon}$ is given by

$$
H_{\varepsilon}(q, b, c)=\frac{1}{2 \varepsilon|\vec{q}|} \exp \left(\varepsilon|\vec{q}| \frac{\sqrt{\Delta}}{a}+\varepsilon q^{0} \frac{c-b}{a}\right)
$$

Proof. Introducing the vector $u=(\varepsilon, \overrightarrow{0})$, we can write the product $f^{\varepsilon} \overline{g^{\varepsilon}}$ as

$$
\left(f^{\varepsilon} \cdot \overline{g^{\varepsilon}}\right)(q)=\int_{0}^{\infty} d c f(c) \int_{0}^{\infty} d b g(b) I(q, b, c)
$$

with

$I(q, b, c)=\int \frac{d^{4} k}{(2 \pi)^{4}} \delta\left(k^{2}-c\right) \delta\left((k-q)^{2}-b\right) \Theta\left(-k^{0}\right) \Theta\left(q^{0}-k^{0}\right) \mathrm{e}^{k u+(k-q) u}$

We choose a reference frame where $q=(0, x, 0,0)$ and $u=(\alpha, \beta, 0,0)$ with $x>0$ and $\alpha>|\beta|$. Choosing cylindrical coordinates $(\omega, p, r, \varphi)$ with $k^{0}=\omega$ 
and $\vec{k}=(p, r \cos \varphi, r \sin \varphi)$, we can compute the integrals,

$$
\begin{aligned}
I(q, b, c)= & \frac{1}{8 \pi^{3}} \int_{-\infty}^{0} d \omega \int_{-\infty}^{\infty} d p \int_{0}^{\infty} r d r \\
& \times \delta\left(\omega^{2}-p^{2}-r^{2}-c\right) \delta\left(\omega^{2}-(p-x)^{2}-r^{2}-b\right) \mathrm{e}^{2 \alpha \omega-\beta(2 p-x)} \\
= & \frac{1}{16 \pi^{3}} \int_{-\infty}^{0} d \omega \int_{-\infty}^{\infty} d p \Theta\left(\omega^{2}-p^{2}-c\right) \\
& \times \delta\left(2 p x-x^{2}+c-b\right) \mathrm{e}^{2 \alpha \omega-\beta(2 p-x)} \\
= & \frac{1}{32 \pi^{3}} \frac{1}{\alpha} \int_{-\infty}^{\infty} d p \delta\left(2 p x-x^{2}+c-b\right) \mathrm{e}^{-2 \alpha \sqrt{p^{2}+c}-\beta(2 p-x)} \\
= & \frac{1}{64 \pi^{3}} \frac{1}{\alpha x} \mathrm{e}^{-2 \alpha \sqrt{K^{2}+c}-\beta(2 K-x)}
\end{aligned}
$$

where we set

$$
K=\frac{x^{2}-c+b}{2 x} .
$$

This can also be written as

$$
I(q, b, c)=\frac{1}{32 \pi^{3}} \frac{1}{2 \alpha x} \exp (-\alpha x A-\beta x B)
$$

where $A$ and $B$ are the expressions

$$
\begin{aligned}
& A=2 \frac{\sqrt{K^{2}+c}}{x}=-\frac{\sqrt{(-a-c+b)^{2}-4 a c}}{a}=-\frac{\sqrt{\Delta}}{a}, \\
& B=\frac{2 K-x}{x}=\frac{c-b}{a}
\end{aligned}
$$

which are covariant and independent of the regularization. Next, we transform back to the original reference frame where $q=\left(q^{0}, \vec{q}\right)$ and $u=(\varepsilon, \overrightarrow{0})$. Since $\beta x=-u q=-\varepsilon q^{0}$ and $\alpha x=\sqrt{-u^{2} q^{2}+(u q)^{2}}=\varepsilon|\vec{q}|$, we have the simple transformation rules

$$
\alpha x \rightarrow \varepsilon|\vec{q}|, \quad \beta x \rightarrow-\varepsilon q^{0} .
$$

Applying these transformation rules in (5.21) gives (5.20). 
Relation (5.19) follows immediately from the transformations

$$
\left(\partial_{k} f^{\varepsilon} \cdot \partial^{k} \overline{g^{\varepsilon}}\right)(q)=\int_{0}^{\infty} d c \int_{0}^{\infty} d b f(c) g(b) K(q, b, c)
$$

with

$$
\begin{aligned}
K(q, b, c)= & \int \frac{d^{4} k}{(2 \pi)^{4}} k_{l}(k-q)^{l} \delta\left(k^{2}-c\right) \delta\left((k-q)^{2}-b\right) \Theta\left(k^{0}\right) \mathrm{e}^{k u+(k-q) u} \\
= & -\int \frac{d^{4} k}{(2 \pi)^{4}} \frac{1}{2}\left((k-(k-q))^{2}-k^{2}-(k-q)^{2}\right) \\
& \times \delta\left(k^{2}-c\right) \delta\left((k-q)^{2}-b\right) \Theta\left(k^{0}\right) \mathrm{e}^{k u+(k-q) u} \\
= & -\frac{1}{2}(a-c-b) I(q, b, c)
\end{aligned}
$$

Obviously, the function $H_{\varepsilon},(5.20)$, becomes singular as $\varepsilon \searrow 0$. The next lemma shows that after dropping suitable terms which are supported on the light cone, the limit $\varepsilon \searrow 0$ exists and is Lorentz invariant.

Lemma 5.4. Suppose that $f\left(k^{2}\right)$ and $g\left(k^{2}\right)$ are negative distributions which vanish identically for large $k^{2}$. Then the products of the corresponding regularized distributions (5.16) have the decomposition

$$
\begin{aligned}
\left(f^{\varepsilon} \cdot \overline{g^{\varepsilon}}\right)(q) & =S_{1}^{\varepsilon}(q)+R_{1}^{\varepsilon}(q), \\
\left(\partial_{k} f^{\varepsilon} \cdot \partial^{k} \overline{g^{\varepsilon}}\right)(q) & =S_{2}^{\varepsilon}(q)+R_{2}^{\varepsilon}(q),
\end{aligned}
$$

where the $S_{i}^{\varepsilon}$ are distributions which are supported on the light cone,

$$
\operatorname{supp} S_{i}^{\varepsilon} \subset\left\{\xi^{2}=0\right\}
$$

and the $R_{i}^{\varepsilon}$ are regular as $\varepsilon \searrow 0$. The limits

$$
R_{i}=\lim _{\varepsilon \searrow 0} R_{i}^{\varepsilon}
$$

are the Lorentz invariant distributions

$$
R_{i}(q)=\int_{0}^{\infty} d c f(c) \int_{0}^{\infty} d b g(b) K_{i}(q, b, c)
$$


with

$$
\begin{aligned}
& K_{1}(q, b, c)=\frac{1}{32 \pi^{3}} \begin{cases}\frac{\sqrt{\Delta}}{a} \Theta(\sqrt{b}-\sqrt{a}-\sqrt{c}) & \text { if } q \in \mathcal{C}^{\vee}, \\
-\frac{|b-c|}{a} \Theta(b-c) & \\
\frac{\sqrt{\Delta} \Theta(\sqrt{c}-\sqrt{a}-\sqrt{b})}{a} & \text { if } q \in \mathcal{C}^{\wedge}, \\
-\frac{|b-c|}{a} \Theta(c-b) & \text { if } q \notin \mathcal{C},\end{cases} \\
& K_{2}(q, b, c)=\frac{1}{32 \pi^{3}}
\end{aligned}
$$

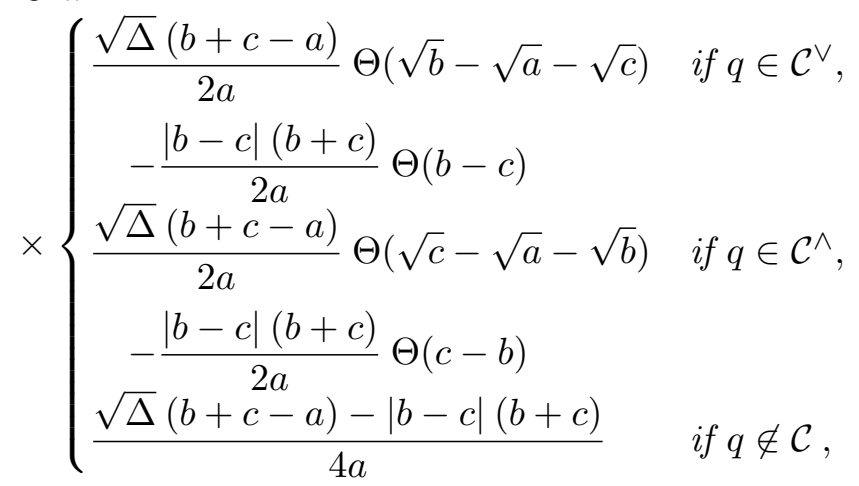

where we again set $a=q^{2}$.

It is interesting to compare the result of this lemma in the case $q \in \mathcal{C}^{\wedge}$ with Lemma 5.2. Then the first summands in (5.24) and (5.25) coincide precisely with (5.11) and (5.14), respectively. The appearance of the additional second summands in (5.24) and (5.25) is somewhat surprising; it shows that extending the convolution integral in the distributional sense to $q \in \hat{M}$ yields additional contributions inside the mass cone. These additional contributions can be understood from the fact they remove the poles of the convolution integral on the cone $\left\{q \mid q^{2}=0\right\}$. Namely, using the expansion

$$
\sqrt{\Delta}=|b-c|+\mathcal{O}(a)
$$

one sees that $K_{1}$ and $K_{2}$ have no poles at $a=0$.

Proof of Lemma 5.4. We first consider the product $f^{\varepsilon} \cdot \overline{g^{\varepsilon}}$. Extending the function $H_{\varepsilon},(5.20)$, by zero to the interior mass cone $q \in \mathcal{C}$, we obtain the 
expansion

$$
H_{\varepsilon}(q, b, c)=\frac{\Theta(-a)}{2 \varepsilon|\vec{q}|}+\frac{q^{0}}{2|\vec{q}|} \frac{\Theta(-a)}{a}(c-b)+\frac{\sqrt{\Delta}}{2 a} \Theta(-a)+\mathcal{O}(\varepsilon)
$$

Here the poles on the mass cone have a mathematical meaning as distributional derivatives of the regular distribution $\Theta(-a) \log a$. We implicitly use this procedure throughout the proof. The first two summands in (5.26) are not Lorentz invariant, and we must analyze their behavior in position space. In preparation, we derive a useful formula for spherically symmetric distributions which are supported on the light cone. In position space, such a distribution can clearly be written in the form $g\left(\xi^{0}\right) \delta\left(\xi^{2}\right)$. We compute its Fourier transform in polar coordinates $x=(t, r, \vartheta, \varphi)$,

$$
\begin{aligned}
f(q) & :=\int d^{4} \xi g\left(\xi^{0}\right) \delta\left(\xi^{2}\right) \mathrm{e}^{-\mathrm{i} q x} \\
& =2 \pi \int_{-\infty}^{\infty} d t g(t) \mathrm{e}^{-\mathrm{i} q^{0} t} \int_{0}^{\infty} r^{2} d r \delta\left(t^{2}-r^{2}\right) \int_{-1}^{1} d \cos \vartheta \mathrm{e}^{\mathrm{i}|\vec{q}| r \cos \vartheta} \\
& =\frac{2 \pi}{i|\vec{q}|} \int_{-\infty}^{\infty} d t g(t) \mathrm{e}^{-\mathrm{i} q^{0} t} \int_{0}^{\infty} r d r \delta\left(t^{2}-r^{2}\right)\left(\mathrm{e}^{\mathrm{i}|\vec{q}| r}-\mathrm{e}^{-\mathrm{i}|\vec{q}| r}\right) \\
& =\frac{\pi}{i|\vec{q}|} \int_{-\infty}^{\infty} d t g(t) \mathrm{e}^{-\mathrm{i} q^{0} t}\left(\mathrm{e}^{\mathrm{i}|\vec{q}||t|}-\mathrm{e}^{-\mathrm{i}|\vec{q}||t|}\right) \\
& =\frac{\pi}{i|\vec{q}|} \int_{-\infty}^{\infty} d t g(t) \epsilon(t) \mathrm{e}^{-\mathrm{i} q^{0} t}\left(\mathrm{e}^{\mathrm{i}|\vec{q}| t}-\mathrm{e}^{-\mathrm{i}|\vec{q}| t}\right) \\
& =\frac{1}{2|\vec{q}|}\left(h\left(q^{0}+|\vec{q}|\right)-h\left(q^{0}-|\vec{q}|\right)\right)
\end{aligned}
$$

where $h$ is the Fourier transform of the function $2 \mathrm{i} \pi g(t) \epsilon(t)$. Since the function $g$ can be arbitrary, the function $h$ is also arbitrary. Also, the above calculation clearly carries through if $h$ is a one-dimensional distribution. We conclude that for any distribution $h$, the expression

$$
f(q)=\frac{1}{|\vec{q}|}\left(h\left(q^{0}+|\vec{q}|\right)-h\left(q^{0}-|\vec{q}|\right)\right)
$$

is supported on the light cone. Choosing $h(x)=\epsilon(x)$, one sees that the distribution $2 \Theta(-a) /|\vec{q}|$, and thus also the first summand in (5.26), are supported on the light cone. The second summand is not of the required 
form. But choosing $h(x)=(c-b) \epsilon(x) /(4 x)$, one sees that its extension

$$
f(q)=\frac{c-b}{2 a} \times \begin{cases}\frac{q^{0}}{|\vec{q}|} & \text { if } q \notin \mathcal{C}, \\ -1 & \text { if } q \in \mathcal{C}^{\vee}, \\ 1 & \text { if } q \in \mathcal{C}^{\wedge}\end{cases}
$$

is supported on the light cone. Setting

$$
S_{\varepsilon}=\frac{\Theta(-a)}{2 \varepsilon|\vec{q}|}+f(q)+\frac{|b-c|}{2} \frac{\mathrm{PP}}{a},
$$

$S_{\varepsilon}$ is supported on the light cone, and the difference $H_{\varepsilon}-S_{\varepsilon}$ has a Lorentz invariant limit,

$$
\lim _{\varepsilon \searrow 0}\left(H_{\varepsilon}-S_{\varepsilon}\right)= \begin{cases}-\frac{|b-c|}{a} \Theta(b-c) & \text { if } q \in \mathcal{C}^{\vee}, \\ -\frac{|b-c|}{a} \Theta(c-b) & \text { if } q \in \mathcal{C}^{\wedge}, \\ \frac{\sqrt{\Delta}-|b-c|}{2 a} & \text { if } q \notin \mathcal{C} .\end{cases}
$$

If $q \in \mathcal{C}^{\wedge}$, we must also take into account the contribution from Lemma 5.2. In the case $q \in \mathcal{C}^{\vee}$, we can apply Lemma 5.2 after double conjugation,

$$
(f \cdot \bar{g})(q)=\overline{(g \cdot \bar{f})(-q)}=\frac{1}{32 \pi^{3}} \int_{a}^{\infty} d c g(c) \int_{0}^{(\sqrt{c}-\sqrt{a})^{2}} d b f(b) \frac{\sqrt{\Delta}}{a} .
$$

Adding all these contributions gives the result for the product $f^{\varepsilon} \cdot \overline{g^{\varepsilon}}$.

In order to treat the product $\partial_{k} f^{\varepsilon} \cdot \partial^{k} \overline{g^{\varepsilon}}$, we note that (5.14) and (5.19) differ from the corresponding formulas (5.11) and (5.18) only by the additional factor $(b+c-a) / 2$. Since $b$ and $c$ were treated as fixed parameters throughout the proof, this additional factor can be written as the differential operator in position space $(b+c+\square \xi) / 2$. Using that the distributions $\square \xi S_{i}^{\varepsilon}$ are again supported on the light cone, the above arguments all go through after applying the operator $(b+c+\square \xi) / 2$. From this consideration we obtain (5.23) with

$$
K_{2}(q, b, c)=K_{1}(q, b, c) \frac{b+c-a}{2} .
$$


Finally, we subtract the contribution

$$
\begin{cases}\frac{|b-c|}{2} \Theta(b-c) & \text { if } q \in \mathcal{C}^{\vee} \\ \frac{|b-c|}{2} \Theta(c-b) & \text { if } q \in \mathcal{C}^{\wedge} \\ \frac{|b-c|}{4} & \text { if } q \notin \mathcal{C}\end{cases}
$$

which for any fixed $b$ and $c$ can be written as $c_{1}+c_{2} \Theta\left(q^{2}\right) \epsilon\left(q^{0}\right)$ with constants $c_{1}$ and $c_{2}$. The Fourier transform of the first summand is a multiple of the distribution $\delta^{4}(\xi)$. Using that $\square_{q}^{2}\left(\Theta\left(q^{2}\right) \epsilon\left(q^{0}\right)\right)=0$ in the distributional sense, one sees that the second summand is supported on the light cone (alternatively, see Corollary 6.1 below). We conclude that contribution (5.28) is supported on the light cone $\left\{\xi \mid \xi^{2}=0\right\}$, completing the proof.

This lemma shows that the products $f \cdot \bar{g}$ and $\partial_{k} f \cdot \partial^{k} \bar{g}$ are singular only on the light cone. Furthermore, it gives a useful explicit formula for the regular contribution away from the light cone in momentum space. Note that the regularization is needed in order to make the singular contribution on the light cone finite, but the remaining regular contribution does not depend on the regularization. With this in mind, we write the result of Lemma 5.4 in the compact form

$$
\begin{aligned}
(f \cdot \bar{g})(q) & =(\text { l.c. })+\int_{0}^{\infty} d c f(c) \int_{0}^{\infty} d b g(b) K_{1}(q, b, c), \\
\left(\partial_{k} f \cdot \partial^{k} \bar{g}\right)(q) & =(\text { l.c. })+\int_{0}^{\infty} d c f(c) \int_{0}^{\infty} d b g(b) K_{2}(q, b, c),
\end{aligned}
$$

where "(l.c.)" stands for a singular (possibly infinite) contribution on the light cone.

The next lemma treats the products which involve one derivative.

Lemma 5.5. Suppose that $f$ and $g$ are negative, Lorentz invariant distributions. Then, using the short notation introduced before (5.29),

$$
\begin{aligned}
& (\not \partial f \cdot \bar{g})(q)=(\text { l.c. })+\int_{0}^{\infty} d c f(c) \int_{0}^{\infty} d b g(b) L_{1}(q, b, c), \\
& (f \cdot \not \partial \bar{g})(q)=(\text { l.c. })+\int_{0}^{\infty} d c f(c) \int_{0}^{\infty} d b g(b) L_{2}(q, b, c)
\end{aligned}
$$


with

$$
\begin{aligned}
& L_{1}(q, b, c)=\frac{\mathrm{i} \not q}{32 \pi^{3}} \\
& \times \begin{cases}\sqrt{\Delta} \frac{a-b+c}{2 a^{2}} \Theta(\sqrt{b}-\sqrt{a}-\sqrt{c}) & \text { if } q \in \mathcal{C}^{\vee}, \\
+\frac{(b-c)^{2}-2 a b}{2 a^{2}} \Theta(b-c) & \text { if } q \in \mathcal{C}^{\wedge}, \\
\sqrt{\Delta} \frac{a-b+c}{2 a^{2}} \Theta(\sqrt{c}-\sqrt{a}-\sqrt{b}) & \\
-\frac{(b-c)^{2}-2 a b}{2 a^{2}} \Theta(c-b) & \text { if } q \notin \mathcal{C}, \\
\sqrt{\Delta} \frac{a-b+c}{4 a^{2}}+\frac{(b-c)^{2}-2 a b}{4 a^{2}} \epsilon(b-c)\end{cases} \\
& L_{2}(q, b, c)=\frac{\mathrm{i} \not q}{32 \pi^{3}} \\
& \times \begin{cases}\sqrt{\Delta} \frac{a+b-c}{2 a^{2}} \Theta(\sqrt{b}-\sqrt{a}-\sqrt{c}) & \text { if } q \in \mathcal{C}^{\vee}, \\
-\frac{(b-c)^{2}-2 a c}{2 a^{2}} \Theta(b-c) & \text { if } q \in \mathcal{C}^{\wedge}, \\
\sqrt{\Delta} \frac{a+b-c}{2 a^{2}} \Theta(\sqrt{c}-\sqrt{a}-\sqrt{b}) & \\
+\frac{(b-c)^{2}-2 a c}{2 a^{2}} \Theta(c-b) & \text { if } q \notin \mathcal{C},\end{cases}
\end{aligned}
$$

where again $a=q^{2}$.

Proof. Using that differentiation in position space corresponds to multiplication in momentum space and vice versa, we obtain from (5.16) that

$$
\begin{aligned}
\not_{\xi} f^{\varepsilon}(k) & =\mathrm{i} \not h f\left(k^{2}\right) \mathrm{e}^{\varepsilon k^{0}}=\frac{\mathrm{i}}{2}\left(\not \not_{k} h\left(k^{2}\right)\right) \mathrm{e}^{\varepsilon k^{0}} \\
& =\frac{\mathrm{i}}{2} \not \not_{k}\left(h\left(k^{2}\right) \mathrm{e}^{\varepsilon k^{0}}\right)-\frac{\mathrm{i} \varepsilon}{2} \gamma^{0} h\left(k^{2}\right) \mathrm{e}^{\varepsilon k^{0}}=\frac{1}{2}\left(-\not{b}-\mathrm{i} \varepsilon \gamma^{0}\right) h^{\varepsilon}(k),
\end{aligned}
$$

where the negative, regularized distribution $h^{\varepsilon}$ is defined by

$$
h^{\varepsilon}(k)=h\left(k^{2}\right) \mathrm{e}^{\varepsilon k^{0}} \quad \text { and } \quad h\left(k^{2}\right)=\int_{0}^{k^{2}} f(e) d e .
$$


Now we can compute the product $\not \partial f \cdot \bar{g}$ with the help of Lemma 5.4,

$$
\begin{aligned}
\not \partial f \cdot \bar{g} & =\lim _{\varepsilon \searrow 0} \frac{1}{2}\left(-\not b-\mathrm{i} \varepsilon \gamma^{0}\right) h^{\varepsilon} \overline{g^{\varepsilon}} \\
& =(\text { l.c. })+\int_{0}^{\infty} d c h(c) \int_{0}^{\infty} d b g(b) \frac{-\not}{2} K_{1}(q, b, c) .
\end{aligned}
$$

Applying the transformations $\sharp=-\mathrm{i} \not \partial_{q}$ and

$$
\begin{aligned}
\int_{0}^{\infty} d c h(c) K_{1}(q, b, c) & =\int_{0}^{\infty} d c \int_{0}^{c} d e f(e) K(q, b, c) \\
& =\int_{0}^{\infty} d e f(e) \int_{e}^{\infty} d c K_{1}(q, b, c),
\end{aligned}
$$

we obtain representation (5.31) with

$$
L_{1}(q, b, e)=\int_{e}^{\infty} \frac{\mathrm{i}}{2} \not_{q} K_{1}(q, b, c) d c .
$$

In order to compute $L_{1}$ more explicitly, we substitute for $K_{1}$ expression (5.24) and carry out the $c$-integration. The following indefinite integrals are easily verified by differentiation

$$
\begin{aligned}
\int \frac{d}{d a} \frac{\sqrt{\Delta}}{a} d c & =\sqrt{\Delta} \frac{b-c-a}{2 a^{2}}, \\
\int \frac{d}{d a} \frac{|b-c|}{a} d c & =|b-c| \frac{b-c}{2 a^{2}} .
\end{aligned}
$$

The asymptotic expansion for large $c$

$$
\begin{aligned}
\sqrt{\Delta} & =(c-b)-a \frac{c+b}{c-b}+\mathcal{O}\left(c^{-2}\right), \\
\sqrt{\Delta}(b-c-a) & =-(c-b)^{2}+2 a b+a^{2}+\mathcal{O}\left(c^{-1}\right)
\end{aligned}
$$

shows that the difference of the two indefinite integrals has a finite limit as $c \rightarrow \infty$,

$$
\lim _{c \rightarrow \infty}\left(\sqrt{\Delta} \frac{b-c-a}{2 a^{2}}-|b-c| \frac{b-c}{2 a^{2}}\right)=\frac{b}{a}+\frac{1}{2} .
$$


Taking into account the boundary conditions as determined by the Heaviside functions in (5.24) gives for $L_{1}$ the expression

$$
\frac{\mathrm{i} \phi}{32 \pi^{3}} \begin{cases}\sqrt{\Delta} \frac{a-b+c}{2 a^{2}} \Theta(\sqrt{b}-\sqrt{a}-\sqrt{c}) & \text { if } q \in \mathcal{C}^{\vee}, \\ & +\frac{(b-c)^{2}}{2 a^{2}} \Theta(b-c) \\ \sqrt{\Delta} \frac{a-b+c}{2 a^{2}} \Theta(\sqrt{c}-\sqrt{a}-\sqrt{b}) & \text { if } q \in \mathcal{C}^{\wedge}, \\ -\frac{(b-c)^{2}}{2 a^{2}} \Theta(c-b)+\frac{b}{a}+\frac{1}{2} & \\ \sqrt{\Delta} \frac{a-b+c}{4 a^{2}}+\frac{|b-c|(b-c)}{4 a^{2}}+\frac{b}{2 a}+\frac{1}{4} & \text { if } q \notin \mathcal{C} .\end{cases}
$$

We finally subtract the distribution

$$
b \Theta(b-c) \frac{\mathrm{PP}}{a}+\frac{1}{4} \Theta(a) \epsilon\left(-q^{0}\right)+\frac{1}{4},
$$

which can be written in the form (5.27) and is therefore localized on the light cone. This gives (5.33).

The formula for $L_{2},(5.34)$, is obtained from (5.33) by double conjugation, $f \cdot \not \partial \bar{g}=\not \partial \bar{g} \cdot \overline{\bar{f}}$. This gives the identity $L_{2}(q, b, c)=L_{1}(-q, c, b)$.

It is instructive to verify the Leibniz rule

$$
\not \partial(f \cdot \bar{g})-(\not \partial f \cdot \bar{g})-(f \cdot \not \partial \bar{g})=0
$$

by substituting the results of Lemmas (5.4) and (5.5). This gives

$$
(\not \partial(f \cdot \bar{g})-(\not \partial f \cdot \bar{g})-(f \cdot \not \partial \bar{g}))(q)=(\text { l.c. })+\int_{0}^{\infty} d c f(c) \int_{0}^{\infty} d b g(b) M(q, b, c)
$$

with

$$
\begin{aligned}
M(q, b, c) & =\mathrm{i} \not q K_{1}(q, b, c)-L_{1}(q, b, c)-L_{2}(q, b, c) \\
& =\frac{\mathrm{i} \phi}{32 \pi^{3}} \begin{cases}-\frac{|b-c|}{a} \Theta(b-c)+\frac{b-c}{2 a} \Theta(b-c) & \text { if } q \in \mathcal{C}^{\vee} \\
-\frac{|b-c|}{a} \Theta(c-b)-\frac{b-c}{a} \Theta(c-b) & \text { if } q \in \mathcal{C}^{\wedge}, \\
-\frac{|b-c|}{2 a}+\frac{b-c}{2 a} \epsilon(b-c) & \text { if } q \notin \mathcal{C} \\
& =0 .\end{cases}
\end{aligned}
$$




\subsection{Convolutions involving Dirac seas}

We now apply the previous results on convolution integrals to composite expressions in the fermionic projector. We begin with the convolution integral (4.10).

Theorem 5.1. For any $k \in \mathcal{C}$ with $k^{2}>0$,

$$
\hat{\mathcal{F}}(k)=\frac{1}{64 \pi^{3}} \frac{\not k}{k^{4}} \epsilon\left(k^{0}\right) \sum_{\alpha, \beta=1}^{g} \rho_{\alpha} \rho_{\beta} J\left(k^{2}, m_{\alpha}, m_{\beta}\right)
$$

where

$$
\begin{aligned}
J(a, x, y)= & -\sqrt{\Delta\left(a, x^{2}, y^{2}\right)}(x-y) \epsilon\left(x^{2}-y^{2}\right) \\
& \times\left[(x+y)^{2}-a\right] \Theta\left((|x|-|y|)^{2}-a\right)
\end{aligned}
$$

with $\Delta$ according to (5.4).

Proof. Introducing the distributions

$$
f(a)=\sum_{\beta=1}^{g} \rho_{\beta} \delta\left(a-m_{\beta}^{2}\right) \quad \text { and } \quad g(a)=\sum_{\beta=1}^{g} \rho_{\beta} m_{\beta} \delta\left(a-m_{\beta}^{2}\right)
$$

we can write the fermionic projector (2.1) with the notation (5.3) as $P=-\mathrm{i} \not \partial f+g$. Then

$$
(P \cdot \bar{P})_{0}=-i((\not \partial f) \cdot \bar{g}-g \cdot(\not \partial \bar{f}))
$$

For $k \in C^{\wedge}$, the appearing convolution integrals were computed in Lemma 5.2. We thus obtain

$$
\begin{aligned}
(P \cdot \bar{P})_{0}= & -\mathrm{i} \not \partial \alpha(a) \\
\alpha(a)= & \frac{1}{32 \pi^{3}} \int_{0}^{\infty} d c f(c) \int_{0}^{\infty} d b g(b) \sqrt{\Delta} \frac{a-b+c}{2 a^{2}} \\
& \times(\Theta(\sqrt{c}-\sqrt{a}-\sqrt{b})-\Theta(\sqrt{b}-\sqrt{a}-\sqrt{c}))
\end{aligned}
$$


where we set $a=k^{2}$. Using (5.37), we can carry out the integrals to obtain (5.35) with

$$
\begin{aligned}
J(a, x, y)= & -2 x \sqrt{\Delta\left(a, x^{2}, y^{2}\right)}\left(a-x^{2}+y^{2}\right)(\Theta(|y|-\sqrt{a}-|x|) \\
& -\Theta(|x|-\sqrt{a}-|y|)) \\
= & -2 x \sqrt{\Delta\left(a, x^{2}, y^{2}\right)}\left(a-x^{2}+y^{2}\right) \epsilon(|y|-|x|) \Theta\left((|y|-|x|)^{2}-a\right) .
\end{aligned}
$$

As (5.35) is symmetric in $\alpha$ and $\beta$, we may symmetrize $J$ in $x$ and $y$, giving (5.36). For $q \in \mathcal{C}^{\vee}$, the result follows similarly using the remark after the proof of Lemma 5.2.

Next we can introduce the distribution $\hat{\mathcal{M}}$, which already appeared in our sketch as the Fourier transform of the distribution $\tilde{\mathcal{M}}$ defined by (4.4). Here we proceed in the opposite way: we first define $\tilde{\mathcal{M}}$ and then show that its Fourier transform really satisfies (4.4). We denote this Fourier transform without a tilde by $\mathcal{M}$. This clarifies that $\mathcal{M}$ is a specific distribution satisfying (4.4), whereas $\tilde{\mathcal{M}}$ is not unique but can be changed freely according to $(4.18)$.

Theorem 5.2. The function

$$
\begin{aligned}
\hat{\mathcal{M}}(k)= & \frac{1}{64 \pi^{3}} \frac{\not k}{k^{4}} \Theta\left(k^{2}\right) \epsilon\left(k^{0}\right) \sum_{\alpha, \beta=1}^{g} \rho_{\alpha} \rho_{\beta} \\
& \times\left(J\left(k^{2}, m_{\alpha}, m_{\beta}\right)+K\left(k^{2}, m_{\alpha}, m_{\beta}\right)\right)
\end{aligned}
$$

with $J$ according to (5.36) and

$$
K(a, x, y)=(x-y)^{2}(x+y)^{3}-2 a\left(x^{3}+y^{3}\right)
$$

defines a tempered distribution, if the pole on the cone $\left\{k \mid k^{2}=0\right\}$ is understood as the distributional derivative of a logarithm, i.e.,

$$
\frac{\not k}{k^{2}} \Theta\left(k^{2}\right) \epsilon\left(k^{0}\right) \quad \text { stands for } \quad \frac{1}{2} \not \not_{k}\left(\log \left(k^{2}\right) \Theta\left(k^{2}\right) \epsilon\left(k^{0}\right)\right) \text {. }
$$

Its Fourier transform $\mathcal{M}(\xi)$ satisfies away from the light cone the relation

$$
\tilde{\mathcal{M}}(\xi)=2 A_{0}(\xi) \quad \forall \xi \notin L
$$

with $A_{0}$ as given by (2.3). 
Proof. It suffices to consider any pair $\alpha, \beta \in\{1, \ldots, g\}$. In the case $m_{\alpha} \neq$ $m_{\beta}$, we write out (5.42) in momentum space to obtain similar to (5.38) the condition

$$
\hat{\mathcal{M}}(k)=-2 \mathrm{i}((\not \partial f) \cdot \bar{g}-g \cdot(\not \partial \bar{f})) .
$$

Computing the convolution integrals with the help of Lemma 5.5 modulo singular contributions on the light cone, a straightforward calculation similar to that in the proof of Theorem 4.1 gives (5.39) with $K$ according to (5.40). Expanding the factor $\sqrt{\Delta}$ in (5.36) for small $a$,

$$
\sqrt{\Delta(a, b, c)}=|b-c|-a \frac{b+c}{|b-c|}+\mathcal{O}\left(a^{2}\right)
$$

one sees that $\hat{\mathcal{M}}(k)$ has no poles on the cone $\left\{k \mid k^{2}=0\right\}$. Thus $\hat{\mathcal{M}}(k)$ is a $L_{\text {loc }}^{1}$-function, which clearly defines a tempered distribution.

In the case $m_{\alpha}=m_{\beta}$, the function $J$ vanishes identically, and thus

$$
\hat{\mathcal{M}}(k)=-\frac{1}{16 \pi^{3}} \frac{\not k}{k^{2}} \epsilon\left(k^{0}\right) \Theta\left(k^{2}\right) \rho_{\alpha} \rho_{\beta} m_{\alpha}^{3} .
$$

Interpreting the pole according to (5.41), this is again a tempered distribution. It has such a simple form that (5.42) can be verified by explicit calculation. Namely, the Fourier transform of $\hat{\mathcal{M}}$ can be given in closed form (see Corollary 6.2 below). Comparing this formula with an explicit computation of $A_{0}$ in position shows that (5.42) is indeed satisfied.

Let us briefly discuss the result of Theorems 5.1 and 5.2. We first point out that, due to the Heaviside function in (5.36), the function $\hat{\mathcal{F}}(k)$ vanishes identically for large $k^{2}$,

$$
\hat{\mathcal{F}}(k)=0 \quad \text { if } k^{2}>\max _{\beta \in\{1, \ldots, g\}} m_{\beta}^{2} .
$$

However, it has poles as $k^{2} \searrow 0$, as one sees from expansion (5.43),

$$
\begin{aligned}
\hat{\mathcal{F}}(k)= & -\frac{\not k \epsilon\left(k^{0}\right)}{64 \pi^{3}} \sum_{\alpha, \beta \mathrm{with} m_{\alpha} \neq m_{\beta}} \rho_{\alpha} \rho_{\beta} \\
& \times\left(\frac{\left(m_{\alpha}-m_{\beta}\right)^{2}\left(m_{\alpha}+m_{\beta}\right)^{3}}{k^{4}}-2 \frac{m_{\alpha}^{3}+m_{\beta}^{3}}{k^{2}}\right) \\
& +\not k \mathcal{O}\left(k^{0}\right) .
\end{aligned}
$$


The distribution $\hat{\mathcal{M}}$ differs from $\hat{\mathcal{F}}$ by the function $K$ in (5.39) and by the fact that the poles of $\hat{\mathcal{M}}$ have a meaning in the distributional sense (5.41). The order of the pole is smaller than that in (5.45),

$$
\begin{aligned}
\hat{\mathcal{M}}(k)= & -\frac{1}{32 \pi^{3}} \not k \cdot \Theta\left(k^{2}\right) \epsilon\left(k^{0}\right) \\
& \times \sum_{\alpha, \beta \text { with } m_{\alpha}=m_{\beta}} \rho_{\alpha} \rho_{\beta} \frac{m_{\alpha}^{3}+m_{\beta}^{3}}{k^{2}}+\not k \mathcal{O}\left(k^{0}\right) .
\end{aligned}
$$

Since (5.35) vanishes identically for large $k^{2}$, using (2.9) and (2.10) we find that $\hat{\mathcal{M}}$ has the simple asymptotics

$$
\hat{\mathcal{M}}(k)=2 \pi^{2} \not k \cdot \Theta\left(k^{2}\right) \epsilon\left(k^{0}\right)\left(\frac{\mathfrak{m}_{3}}{k^{2}}+\frac{4 \mathfrak{m}_{5}}{k^{4}}\right) \quad \text { if } k^{2}>\max _{\beta \in\{1, \ldots, g\}} m_{\beta}^{2} .
$$

We end this section by specifying the effect of the parameters $c_{0}$ and $c_{1}$ in (4.18) on the integral in (4.16).

Lemma 5.6. The Fourier transforms of the distributions

$$
A(\xi):=\not \delta\left(\xi^{2}\right) \epsilon\left(\xi^{0}\right), \quad B(\xi):=\not \delta^{\prime}\left(\xi^{2}\right) \epsilon\left(\xi^{0}\right)
$$

satisfy the relations

$$
\begin{aligned}
& \int_{0}^{\infty} \operatorname{Tr}((\hat{A} * \hat{P})(q) \delta \hat{P}(q)) a d a=32 \pi^{4} \delta \mathfrak{m}_{3}, \\
& \int_{0}^{\infty} \operatorname{Tr}((\hat{B} * \hat{P})(q) \delta \hat{P}(q)) a d a=-32 \pi^{4} \delta \mathfrak{m}_{5},
\end{aligned}
$$

where $\mathfrak{m}_{3}$ and $\mathfrak{m}_{5}$ are defined by $(2.9,2.10)$, and where again $a=q^{2}$.

Proof. Again using notation (5.3), we write the fermionic projector as $\hat{P}=$ $-\mathrm{i} \not \partial f+g$ with $f$ and $g$ according to (5.37). The Fourier transforms of $A$ and $B$ can be computed explicitly (see Corollary 6.1 below). Decomposing them into a sum of a positive and a negative distribution, we write $\hat{A}$ and $\hat{B}$ as

$$
\mathrm{i} \not \partial(h(a)-\bar{h}(a))
$$

with

$$
h(a)=8 \pi^{2} \delta^{\prime}(a) \text { for } \hat{A} \text { and } h(a)=2 \pi^{2} \delta(a) \text { for } \hat{B} .
$$


The convolutions $\hat{P} * \hat{A}$ and $\hat{P} * \hat{B}$ are then expressed by

$$
(\not \partial f+\mathrm{i} g) \cdot(\not \partial h-\not \partial \bar{h}) .
$$

For any $q \in \mathcal{C}^{\wedge}$, we can write these convolutions with the help of Lemmas 5.1 and 5.2 ,

$$
\begin{aligned}
(\not \partial f \cdot(\not \partial h-\not \partial \bar{h}))(a)= & \alpha(a), \\
\alpha(a)= & \frac{1}{32 \pi^{3}} \int_{0}^{\infty} d c f(c) \epsilon(a-c) \int_{0}^{(\sqrt{a}-\sqrt{c})^{2}} \\
& \times d b h(b) \sqrt{\Delta} \frac{c+b-a}{2 a}, \\
(\mathrm{i} g \cdot(\not \partial h-\not \partial \bar{h}))(a)= & \mathrm{i} \not \partial \beta(a), \\
\beta(a)= & \frac{1}{32 \pi^{3}} \int_{0}^{\infty} d c g(c) \epsilon(a-c) \int_{0}^{(\sqrt{a}-\sqrt{c})^{2}} \\
& \times d b h(b) \sqrt{\Delta} \frac{a+b-c}{2 a^{2}} .
\end{aligned}
$$

We now compute the remaining integrals using the special form of the functions $f, g$ and $h$. We begin with the calculation for $\hat{A}$. Using similar to (5.43) the expansion

$$
\sqrt{\Delta}=|a-c|-b \frac{a+c}{|a-c|}+\mathcal{O}\left(b^{2}\right),
$$

we obtain

$$
\begin{aligned}
\alpha(a) & =-\left.\frac{1}{4 \pi} \int_{0}^{\infty} d c f(c) \epsilon(a-c) \frac{d}{d b}\left(\sqrt{\Delta} \frac{c+b-a}{2 a}\right)\right|_{b=0} \\
& =-\frac{1}{4 \pi} \int_{0}^{\infty} d c f(c)=-\frac{1}{4 \pi} \sum_{\alpha=1}^{g} \rho_{\alpha}, \\
\beta(a) & =-\left.\frac{1}{4 \pi} \int_{0}^{\infty} d c g(c) \epsilon(a-c) \frac{d}{d b}\left(\sqrt{\Delta} \frac{a+b-c}{2 a^{2}}\right)\right|_{b=0} \\
& =\frac{1}{4 \pi} \int_{0}^{\infty} d c g(c) \frac{c}{a^{2}}=\frac{1}{4 \pi} \sum_{\alpha=1}^{g} \rho_{\alpha} \frac{m_{\alpha}^{3}}{a^{2}}
\end{aligned}
$$

and thus

$$
(\hat{P} * \hat{A})(q)=-\frac{1}{4 \pi} \sum_{\alpha=1}^{g} \frac{\rho_{\alpha}}{a^{2}}\left(m_{\alpha} \not q+a\right)
$$

Now we take the trace with a "test Dirac sea"

$$
\hat{R}(q):=\rho(\not 1+m) \delta\left(q^{2}-m^{2}\right) .
$$


This gives

$$
\int_{0}^{\infty} \operatorname{Tr}((\hat{P} * \hat{A})(q) \hat{R}(q)) a d a=-\frac{1}{\pi} \sum_{\alpha=1}^{g} \rho \rho_{\alpha}\left(m_{\alpha}^{3}+m^{3}\right) .
$$

After varying $\rho$ and $m$, we can build up $\delta \hat{P}$ by taking the sum of $g$ test Dirac seas. Using the symmetry of (5.51) in $\rho, \rho_{\alpha}$ and $m, m_{\alpha}$, we thus obtain

$$
\int_{0}^{\infty} \operatorname{Tr}((\hat{P} * \hat{A})(q) \delta \hat{P}(q)) a d a=-\frac{1}{2 \pi} \delta\left(\sum_{\alpha=1}^{g} \rho_{\alpha} \rho_{\beta}\left(m_{\alpha}^{3}+m_{\beta}^{3}\right)\right),
$$

and comparing with (2.9) gives (5.48).

The calculation for $\hat{B}$ is similar. Using (5.50), we obtain

$$
\begin{aligned}
\alpha(a) & =\left.\frac{1}{16 \pi} \int_{0}^{\infty} d c f(c) \epsilon(a-c) \sqrt{\Delta} \frac{c+b-a}{2 a}\right|_{b=0} \\
& =-\frac{1}{16 \pi} \int_{0}^{\infty} d c f(c) \frac{(c-a)^{2}}{2 a}=-\frac{1}{32 \pi} \sum_{\alpha=1}^{g} \rho_{\alpha} \frac{\left(m_{\alpha}^{2}-a\right)^{2}}{a}, \\
\beta(a) & =\frac{1}{16 \pi} \int_{0}^{\infty} d c g(c) \frac{(c-a)^{2}}{2 a^{2}}=\frac{1}{32 \pi} \sum_{\alpha=1}^{g} \rho_{\alpha} \frac{m_{\alpha}\left(m_{\alpha}^{2}-a\right)^{2}}{a^{2}}
\end{aligned}
$$

and thus

$$
\begin{aligned}
(\hat{P} * \hat{B})(q) & =-\frac{1}{32 \pi} \sum_{\alpha=1}^{g} \rho_{\alpha} \frac{\left(m_{\alpha}^{2}-a\right)^{2}}{a^{2}}\left(m_{\alpha} \not q+a\right), \\
\int_{0}^{\infty} \operatorname{Tr}((\hat{P} * \hat{B})(q) \hat{R}(q)) a d a & =-\frac{1}{8 \pi} \sum_{\alpha=1}^{g} \rho \rho_{\alpha}\left(m_{\alpha}-m\right)^{2}\left(m_{\alpha}+m\right)^{3} .
\end{aligned}
$$

Comparing with (2.10) gives (5.49).

\section{A Lorentz invariant regularization and its fourier transform}

According to (4.4) and (2.15), (2.8), on the light cone $\tilde{\mathcal{M}}$ has the leading pole

$$
\tilde{\mathcal{M}}(\xi)=\mathfrak{m}_{3} \frac{\not}{\xi^{4}} \Theta\left(\xi^{2}\right) \epsilon\left(\xi^{0}\right)+\mathcal{O}\left(\xi^{-2}\right)
$$

and this pole needs to be regularized in order to give the action a mathematical meaning. In this section we shall construct a Lorentz invariant 
regularization of the pole in (6.1), which is explicit both in position and in momentum space. Our analysis is based on the following Fourier integral.

Lemma 6.1. The following identity holds in the sense of distributions:

$$
\begin{aligned}
\int \frac{d^{4} k}{(2 \pi)^{4}} \mathrm{e}^{-\frac{\varepsilon k^{2}}{2}} \Theta\left(k^{2}\right) \epsilon\left(k^{0}\right) \mathrm{e}^{\mathrm{i} k \xi}= & -\frac{\mathrm{i}}{4 \pi^{2} \varepsilon^{2}} \mathrm{e}^{-\frac{\xi^{2}}{2 \varepsilon}} \Theta\left(\xi^{2}\right) \epsilon\left(\xi^{0}\right) \\
& +\frac{\mathrm{i}}{2 \pi^{2} \varepsilon} \delta\left(\xi^{2}\right) \epsilon\left(\xi^{0}\right) .
\end{aligned}
$$

Proof. Due to spherical symmetry, we can assume that $\xi=(t, r, 0,0)$ with $r \geq 0$. Choosing polar coordinates $k=(\omega, p \cos \vartheta, p \sin \vartheta \cos \varphi, p \sin \vartheta \sin \varphi)$, the Fourier integral becomes

$$
\begin{aligned}
A & :=\int \frac{d^{4} k}{(2 \pi)^{4}} \mathrm{e}^{-\frac{\varepsilon k^{2}}{2}} \Theta\left(k^{2}\right) \epsilon\left(k^{0}\right) \mathrm{e}^{\mathrm{i} k \xi} \\
& =\frac{1}{8 \pi^{3}} \int_{-\infty}^{\infty} d \omega \epsilon(\omega) \mathrm{e}^{\mathrm{i} \omega t} \int_{0}^{|\omega|} p^{2} d p \mathrm{e}^{-\frac{\varepsilon}{2}\left(\omega^{2}-p^{2}\right)} \int_{-1}^{1} d \cos \vartheta \mathrm{e}^{-\mathrm{i} p r \cos \vartheta} \\
& =\frac{\mathrm{i}}{8 \pi^{3} r} \int_{-\infty}^{\infty} d \omega \epsilon(\omega) \mathrm{e}^{\mathrm{i} \omega t} \int_{0}^{|\omega|} p d p \mathrm{e}^{-\frac{\varepsilon}{2}\left(\omega^{2}-p^{2}\right)}\left(\mathrm{e}^{-\mathrm{i} p r}-\mathrm{e}^{\mathrm{i} p r}\right) \\
& =\frac{\mathrm{i}}{8 \pi^{3} r} \int_{-\infty}^{\infty} d \omega \epsilon(\omega) \int_{-|\omega|}^{|\omega|} p d p \mathrm{e}^{-\frac{\varepsilon}{2}\left(\omega^{2}-p^{2}\right)+\mathrm{i}(\omega t-p r)}
\end{aligned}
$$

We now introduce the "mass cone coordinates"

$$
u=\frac{1}{2}(\omega+p), \quad v=\frac{1}{2}(\omega-p),
$$

and light cone coordinates

$$
s=\frac{1}{2}(t-r), \quad l=\frac{1}{2}(t+r) .
$$

Then our integrals transform to

$$
A=\frac{\mathrm{i}}{4 \pi^{3} r}\left(\int_{0}^{\infty} \int_{0}^{\infty}-\int_{-\infty}^{0} \int_{-\infty}^{0}\right) d u d v(u-v) \mathrm{e}^{-2 \varepsilon u v+2 \mathrm{i} u s+2 \mathrm{i} v l}
$$

Carrying out the $v$-integral in the case $u>0$ gives

$$
\begin{gathered}
\int_{0}^{\infty}(u-v) \mathrm{e}^{-2 \varepsilon u v+2 \mathrm{i} v l} d v=\left(u+\frac{\mathrm{i}}{2} \frac{\partial}{\partial l}\right) \\
\int_{0}^{\infty} \mathrm{e}^{-2 \varepsilon u v+2 \mathrm{i} v l} d v=\left(u+\frac{\mathrm{i}}{2} \frac{\partial}{\partial l}\right) \frac{1}{2 \varepsilon u-2 \mathrm{i} l},
\end{gathered}
$$


whereas in the case $u<0$ we get the same expression with the opposite sign. Hence

$$
\begin{aligned}
A & =\frac{\mathrm{i}}{4 \pi^{3} r} \int_{-\infty}^{\infty}\left[\left(u+\frac{\mathrm{i}}{2} \frac{\partial}{\partial l}\right) \frac{1}{2 \varepsilon u-2 \mathrm{i} l}\right] \mathrm{e}^{2 \mathrm{i} u s} d u \\
& =\frac{1}{8 \pi^{3} r}\left(\frac{\partial}{\partial s}-\frac{\partial}{\partial l}\right) \int_{-\infty}^{\infty} \frac{1}{2 \varepsilon u-2 \mathrm{i} l} \mathrm{e}^{2 \mathrm{i} u s} d u \\
& =\frac{\mathrm{i}}{8 \pi^{2} r \varepsilon}\left(\frac{\partial}{\partial s}-\frac{\partial}{\partial l}\right)\left[\epsilon(s) \Theta(s l) \mathrm{e}^{-\frac{2 s l}{\varepsilon}}\right],
\end{aligned}
$$

where in the last step we computed the integral with residues. We finally transform back to polar coordinates

$$
A=-\frac{\mathrm{i}}{4 \pi^{2} \varepsilon} \frac{1}{r} \frac{\partial}{\partial r}\left[\epsilon(t) \Theta\left(t^{2}-r^{2}\right) \mathrm{e}^{-\frac{t^{2}-r^{2}}{2 \varepsilon}}\right],
$$

carry out the distributional derivatives and write the result in Lorentz invariant form.

Before going on, we collect of a few simple Fourier integrals which can easily be obtained from (6.2).

Corollary 6.1. The following equations hold in the sense of distributions:

$$
\begin{gathered}
\int \delta^{\prime}\left(\xi^{2}\right) \epsilon\left(\xi^{0}\right) \mathrm{e}^{-\mathrm{i} k \xi} d^{4} \xi=-\mathrm{i} \pi^{2} \Theta\left(k^{2}\right) \epsilon\left(k^{0}\right), \\
\int \delta\left(\xi^{2}\right) \epsilon\left(\xi^{0}\right) \mathrm{e}^{-\mathrm{i} k \xi} d^{4} \xi=-4 \mathrm{i} \pi^{2} \delta\left(k^{2}\right) \epsilon\left(k^{0}\right), \\
\int \not \delta^{\prime}\left(\xi^{2}\right) \epsilon\left(\xi^{0}\right) \mathrm{e}^{-\mathrm{i} k \xi} d^{4} \xi=2 \pi^{2} \not k \delta\left(k^{2}\right) \epsilon\left(k^{0}\right), \\
\int \not \& \delta\left(\xi^{2}\right) \epsilon\left(\xi^{0}\right) \mathrm{e}^{-\mathrm{i} k \xi} d^{4} \xi=8 \pi^{2} \not k \delta^{\prime}\left(k^{2}\right) \epsilon\left(k^{0}\right) .
\end{gathered}
$$

Proof. Identity (6.3) could be obtained by a direct computation of the Fourier integrals. We here use another method where we recover (6.3) as a limiting case of Lemma 6.1. Using that

$$
\frac{1}{\varepsilon^{2}} \int_{0}^{\infty} \mathrm{e}^{-\frac{z}{2 \varepsilon}} d z-\frac{2}{\varepsilon}=0, \quad \frac{1}{\varepsilon^{2}} \int_{0}^{\infty} z \mathrm{e}^{-\frac{z}{2 \varepsilon}} d z=4
$$

one sees that

$$
\frac{1}{\varepsilon^{2}} \mathrm{e}^{-\frac{z}{2 \varepsilon}} \Theta(z)-\frac{1}{2 \varepsilon} \delta(z) \stackrel{\varepsilon \rightarrow 0}{\longrightarrow}-4 \delta^{\prime}(z)
$$


with convergence in $\mathcal{S}^{\prime}(\mathbb{R})$. This also shows that (6.2) converges in $\mathcal{S}^{\prime}(\hat{M})$ to

$$
\int \frac{d^{4} k}{(2 \pi)^{4}} \Theta\left(k^{2}\right) \epsilon\left(k^{0}\right) \mathrm{e}^{\mathrm{i} k \xi}=\frac{\mathrm{i}}{\pi^{2}} \delta^{\prime}\left(\xi^{2}\right) \epsilon\left(\xi^{0}\right),
$$

except maybe at the origin $\xi=0$, where the light cone has its cusp. To prove convergence at the origin, one verifies easily by a scaling argument that for any test function $\eta \in C_{0}^{\infty}(M)$,

$$
\lim _{\delta \searrow 0} \lim _{\varepsilon \searrow 0} \int \eta\left(\frac{\xi}{\delta}\right)\left[\frac{1}{\varepsilon^{2}} \mathrm{e}^{-\frac{\xi^{2}}{2 \varepsilon}} \Theta\left(\xi^{2}\right) \epsilon\left(\xi^{0}\right)-\frac{1}{2 \varepsilon} \delta\left(\xi^{2}\right) \epsilon\left(\xi^{0}\right)\right]=0
$$

We finally apply Plancherel's theorem to (6.7) to obtain (6.3).

Applying the operator $\square_{k}$ to (6.3) gives (6.4). To derive (6.5), we apply the operator $i \not_{k}$ to (6.3). Finally, identity (6.6) follows from (6.4) by applying the differential operator $i \not_{k}$.

The main importance of Lemma 6.1 lies in the fact that integrating over $\varepsilon$ gives the Fourier transformations of regularized poles.

Proposition 6.1. The following equations hold in the sense of distributions:

$$
\begin{aligned}
& \int \frac{d^{4} k}{(2 \pi)^{4}} \mathrm{e}^{-\frac{\varepsilon k^{2}}{2}} \square_{k}\left(\log \left(k^{2}\right) \Theta\left(k^{2}\right) \epsilon\left(k^{0}\right)\right) \mathrm{e}^{\mathrm{i} k \xi} \\
& =-\frac{\mathrm{i}}{\pi^{2}}\left[\frac{1}{\xi^{2}}\left(1-\mathrm{e}^{-\frac{\xi^{2}}{2 \varepsilon}}\right) \Theta\left(\xi^{2}\right) \epsilon\left(\xi^{0}\right)+\delta\left(\xi^{2}\right) \epsilon\left(\xi^{0}\right)(c-1+\log \varepsilon)\right], \\
& \int \frac{d^{4} k}{(2 \pi)^{4}} \mathrm{e}^{-\frac{\varepsilon k^{2}}{2}} \not_{k}\left(\log \left(k^{2}\right) \Theta\left(k^{2}\right) \epsilon\left(k^{0}\right)\right) \mathrm{e}^{\mathrm{i} k \xi} \\
& \quad=-\frac{1}{2 \pi^{2}} \not \not_{\xi}\left[\frac{1}{\xi^{2}}\left(1-\mathrm{e}^{-\frac{\xi^{2}}{2 \varepsilon}}\right) \Theta\left(\xi^{2}\right) \epsilon\left(\xi^{0}\right)+\delta\left(\xi^{2}\right) \epsilon\left(\xi^{0}\right)(c+\log \varepsilon)\right] .
\end{aligned}
$$

Here the constant $c$ is given by

$$
c=\gamma-\log 2,
$$

and $\gamma$ is Euler's constant. 
Proof. Integrating the variable $\varepsilon$ in $(6.2)$ over the compact interval $[\varepsilon, L]$ with $L>\varepsilon$ gives the identity

$$
\begin{aligned}
& \int \frac{d^{4} k}{(2 \pi)^{4}} \frac{2}{k^{2}}\left(\mathrm{e}^{-\frac{\varepsilon k^{2}}{2}}-\mathrm{e}^{-\frac{L k^{2}}{2}}\right) \Theta\left(k^{2}\right) \epsilon\left(k^{0}\right) \mathrm{e}^{\mathrm{i} k \xi} \\
& =-\frac{\mathrm{i}}{4 \pi^{2}} \frac{2}{\xi^{2}}\left(\mathrm{e}^{-\frac{k^{2}}{2 L}}-\mathrm{e}^{-\frac{\xi^{2}}{2 \varepsilon}}\right) \Theta\left(\xi^{2}\right) \epsilon\left(\xi^{0}\right)+\frac{\mathrm{i}}{2 \pi^{2}} \delta\left(\xi^{2}\right) \epsilon\left(\xi^{0}\right)(\log L-\log \varepsilon) .
\end{aligned}
$$

Bringing the term involving $\log L$ to the left, we can use (6.4) to combine it with the integrand,

$$
\begin{gathered}
\int \frac{d^{4} k}{(2 \pi)^{4}}\left\{\frac{1}{k^{2}}\left(\mathrm{e}^{-\frac{\varepsilon k^{2}}{2}}-\mathrm{e}^{-\frac{L k^{2}}{2}}\right) \Theta\left(k^{2}\right) \epsilon\left(k^{0}\right)-\delta\left(k^{2}\right) \epsilon\left(k^{0}\right)(c+\log L)\right\} \mathrm{e}^{\mathrm{i} k \xi} \\
=-\frac{\mathrm{i}}{4 \pi^{2}}\left[\frac{1}{\xi^{2}}\left(\mathrm{e}^{-\frac{k^{2}}{2 L}}-\mathrm{e}^{-\frac{\xi^{2}}{2 \varepsilon}}\right) \Theta\left(\xi^{2}\right) \epsilon\left(\xi^{0}\right)+\delta\left(\xi^{2}\right) \epsilon\left(\xi^{0}\right)(c+\log \varepsilon)\right] .
\end{gathered}
$$

A direct calculation yields for any $\eta \in C_{0}^{\infty}(\mathbb{R})$ and $x>0$ the relations

$$
\begin{aligned}
& \lim _{L \rightarrow \infty}\left(\int_{-\infty}^{x} \eta(a) \frac{1}{a}\left(\left(1-\mathrm{e}^{-\frac{L a}{2}}\right) \Theta(a)\right) d a-\log L\right) \\
& \quad=\eta(0)(\log (x)+\gamma-\log 2)+\mathcal{O}(x) \\
& \int_{-\infty}^{x} \eta(a) \frac{1}{a} \frac{d}{d a}\left(a^{2} \frac{d}{d a}(\Theta(a) \log a)\right)=\eta(0)(\log (x)+1)+\mathcal{O}(x) .
\end{aligned}
$$

Thus, choosing $c$ according to (6.11), we obtain

$$
\begin{aligned}
& \lim _{L \rightarrow \infty}\left\{\frac{1}{a}\left(\mathrm{e}^{-\frac{\varepsilon a}{2}}-\mathrm{e}^{-\frac{L a}{2}}\right) \Theta(a)-\delta(a)(c-1+\log L)\right\} \\
& =-\frac{1}{4} \hat{W}(\log (a) \Theta(a)) \mathrm{e}^{-\frac{\varepsilon a}{2}}
\end{aligned}
$$

where $\hat{W}$ is the differential operator (3.15), and the derivatives and the convergence are meant in $\mathcal{S}^{\prime}(\mathbb{R})$. Using at the origin a scaling argument similar to (6.8), we can thus take the limit $L \rightarrow \infty$ on the left of (6.12) to obtain (6.9). 
In order to derive (6.10), we apply the operator $-\mathrm{i} \not \partial_{\xi}$ to (6.12). Using (6.13) together with the identity

$$
\int_{-\infty}^{x} \eta(a) \frac{d}{d a}(\Theta(a) \log a)=\eta(0) \log (x)+o(x),
$$

we conclude that

$$
\begin{aligned}
& \lim _{L \rightarrow \infty}\left\{\frac{\not k}{a}\left(\mathrm{e}^{-\frac{\varepsilon a}{2}}-\mathrm{e}^{-\frac{L a}{2}}\right) \Theta(a)-\delta(a)(c+\log L)\right\} \epsilon\left(k^{0}\right) \\
& \quad=\frac{1}{2} \not \not_{k}\left(\log (a) \Theta(a) \epsilon\left(k^{0}\right)\right) \mathrm{e}^{-\frac{\varepsilon a}{2}},
\end{aligned}
$$

giving (6.10).

The method that we just used to take the limit $L \rightarrow \infty$ can be also be applied to take the limit $\varepsilon \searrow 0$ in (6.9) and (6.10), giving the following result.

Corollary 6.2. The following equations hold in the sense of distributions:

$$
\begin{aligned}
& \int \frac{d^{4} k}{(2 \pi)^{4}} \square_{k}\left(\log \left(k^{2}\right) \Theta\left(k^{2}\right) \epsilon\left(k^{0}\right)\right) \mathrm{e}^{\mathrm{i} k \xi} \\
& =-\frac{\mathrm{i}}{4 \pi^{2}} \square_{\xi}\left(\log \left(\xi^{2}\right) \Theta\left(\xi^{2}\right) \epsilon\left(\xi^{0}\right)\right), \\
& \int \frac{d^{4} k}{(2 \pi)^{4}} \not \partial_{k}\left(\log \left(k^{2}\right) \Theta\left(k^{2}\right) \epsilon\left(k^{0}\right)\right) \mathrm{e}^{\mathrm{i} k \xi} \\
& =-\frac{1}{8 \pi^{2}} \not \partial_{\xi} \square_{\xi}\left(\left(\log \left(\xi^{2}\right)-1\right) \Theta\left(\xi^{2}\right) \epsilon\left(\xi^{0}\right)\right) .
\end{aligned}
$$

Let us explain why the previous proposition is so useful for regularizing $\tilde{\mathcal{M}}$ near the light cone. Our goal is to introduce a regularization $\tilde{\mathcal{M}}^{\varepsilon}$ which is explicit both in position and momentum space and makes our action finite, i.e.,

$$
\int_{0}^{\infty}\left(\tilde{\mathcal{M}}^{\varepsilon}\right)^{2} a d a<\infty
$$

(see also (4.1) and the discussion thereafter). Carrying out the derivatives, one sees that distribution (6.16) has a pole of the desired form (6.1),

$$
(6.16)=\frac{2 \mathrm{i}}{\pi^{2}} \frac{\not}{\xi^{4}} \Theta\left(\xi^{2}\right) \epsilon\left(\xi^{0}\right) \quad \text { if } \xi \notin L .
$$


Thus (6.16) extends this pole in the distributional sense across the light cone and gives an explicit formula for the corresponding Fourier transform. But this does clearly not solve the problem of making integral (6.17) finite. The integrand in (6.10) differs from that in (6.16) by the factor $\mathrm{e}^{-\frac{\varepsilon k^{2}}{2}}$. The decay of this factor for large $k^{2}$ can be thought of as a "smooth momentum cutoff," and thus we refer to it as the

$$
\text { regularizing factor } \mathrm{e}^{-\frac{\varepsilon k^{2}}{2}} \text { in momentum space . }
$$

The interesting point is the effect of this regularization on the pole in position space as described by the right side of (6.10). The term $\delta\left(\xi^{2}\right)$ leads to a distributional contribution supported on the light cone. It is not obvious how to make sense of it in an integral of the form (6.17). But we can simply drop this contribution by considering instead of (6.17) an integral of the form

$$
\int_{0_{+}}^{\infty}\left(\tilde{\mathcal{M}}^{\varepsilon}\right)^{2} a d a:=\lim _{\delta \searrow 0} \int_{\delta}^{\infty}\left(\tilde{\mathcal{M}}^{\varepsilon}\right)^{2} a d a
$$

(we remark that an equivalent, but maybe a bit cleaner method for getting rid of the distribution supported at $a=0$ is to subtract this distribution from (6.17) and to use that, according to (6.5), this changes the Fourier transform only by an irrelevant contribution supported on the mass cone $\left.\left\{k \mid k^{2}=0\right\}\right)$. Disregarding the $\delta$-distribution, the effect of the regularization can be described by the

$$
\text { replacement } \frac{1}{z^{2}} \longrightarrow-\frac{d}{d z}\left[\frac{1}{z}\left(1-e^{-\frac{z}{2 \varepsilon}}\right)\right] \text { in position space. }
$$

Using the formula

$$
\frac{1}{z}\left(1-\mathrm{e}^{-\frac{z}{2 \varepsilon}}\right)=\int_{0}^{\frac{1}{2 \varepsilon}} \mathrm{e}^{-\lambda z} d \lambda
$$

one sees that the square bracket in (6.20) is a smooth function in the variable $z$. Also, due to the decay of the factor $\mathrm{e}^{-\frac{z}{2 \varepsilon}}$, the regularization is indeed localized in a strip $z \sim \varepsilon$ around the light cone. With (6.18) and (6.20) we have a simple regularization method, which is convenient both in momentum and position space.

We are now ready to complete the constructions outlined in Section 4 . According to the left of (6.16), the pole $\sim \$ / \xi^{4}$ corresponds in momentum space to a term of the form $\not k / k^{2}$. Thus the relevant term for the regularization of $\hat{\mathcal{M}}$ can be read off from expansion (5.47). 
Definition 6.1. For any $\varepsilon>0$ and $k \in \mathcal{C}$ with $k^{2}>0$, we introduce the function $\hat{\mathcal{N}}^{\varepsilon}$ by

$$
\begin{aligned}
\hat{\mathcal{N}}^{\varepsilon}(k)= & \frac{1}{64 \pi^{3}} \frac{\not k}{k^{4}} \epsilon\left(k^{0}\right) \sum_{\alpha, \beta=1}^{g} \rho_{\alpha} \rho_{\beta} K\left(k^{2}, m_{\alpha}, m_{\beta}\right) \\
& +2 \pi^{2} \mathfrak{m}_{3} \frac{\not k}{k^{2}}\left(\mathrm{e}^{-\frac{\varepsilon k^{2}}{2}}-1\right) \epsilon\left(k^{0}\right),
\end{aligned}
$$

where $K$ is function (5.40). For any $\varepsilon>0$, we define the distribution $\hat{\mathcal{M}}^{\varepsilon}$ by

$$
\hat{\mathcal{M}}^{\varepsilon}(k)=\hat{\mathcal{M}}(k)+2 \pi^{2} \mathfrak{m}_{3} \frac{\not k}{k^{2}}\left(\mathrm{e}^{-\frac{\varepsilon k^{2}}{2}}-1\right) \Theta\left(k^{2}\right) \epsilon\left(k^{0}\right)
$$

with $\hat{\mathcal{M}}$ according to (5.39). The Fourier transform of $\hat{\mathcal{M}}^{\varepsilon}$ is denoted by $\mathcal{M}^{\varepsilon}$.

Proof of Lemma 4.1. From expansion (5.46) we know that for any $\varepsilon>0$, the pole of $\hat{\mathcal{M}}^{\varepsilon}(k)$ on the mass cone is integrable in the $L^{2}(\hat{M}, a d a)$-norm. Furthermore, from (5.47) and Definition 6.1 we see that $\mathcal{M}^{\varepsilon}(k)$ for large $k^{2}$ has the form

$$
\hat{\mathcal{M}}^{\varepsilon}(k)=2 \pi^{2} \not k \Theta\left(k^{2}\right) \epsilon\left(k^{0}\right)\left(\frac{\mathfrak{m}_{3}}{k^{2}} \mathrm{e}^{-\frac{\varepsilon k^{2}}{2}}+\frac{4 \mathfrak{m}_{5}}{k^{4}}\right) \quad \text { if } k^{2}>\max _{\beta \in\{1, \ldots, g\}} m_{\beta}^{2} .
$$

We conclude that $\hat{\mathcal{M}}^{\varepsilon} \in L^{2}(\hat{M}, a d a)$, and thus Corollary 3.1 applies

$$
\int_{0_{+}} \operatorname{Tr}\left(\left(\mathcal{M}^{\varepsilon}\right)^{2}\right) z d z=\frac{1}{(2 \pi)^{4}} \int_{0_{+}} \operatorname{Tr}\left(\left(\hat{\mathcal{M}}^{\varepsilon}\right)^{2}\right) a d a
$$

were we disregard the distributional contributions at $z=0$ and $a=0$ as explained before (6.19). From (6.22) we can also determine the counter term needed in (4.7). Namely, substituting (6.22) into (4.7) and computing the integral, we find that choosing

$$
F_{\varepsilon}\left(\mathfrak{m}_{3}, \mathfrak{m}_{5}\right)=G\left(\mathfrak{m}_{3}, \mathfrak{m}_{5}\right)-\frac{4 \pi^{4} \mathfrak{m}_{3}^{2}}{\varepsilon}-32 \pi^{4} \mathfrak{m}_{3} \mathfrak{m}_{5} \log \varepsilon
$$

the limit $\varepsilon \searrow 0$ exist. The limit clearly coincides with that in (2.12) for a suitable choice of the function $F-G$.

Identity (4.9) follows immediately from Definition 6.1, and thus it remains to justify the limits in $(4.11)$ and $(4.12)$. Since $\hat{\mathcal{F}}$ vanishes for large $k^{2}$ according to (5.44), the integral clearly converges for large $a$. For the behavior 
near $a=0$, we use expansion (5.45) to rewrite the integral in (4.11) as

$$
\int_{0}^{\infty}\left[\frac{\not k}{a} \hat{\mathcal{M}}^{\varepsilon}\right](c+\mathcal{O}(a)) d a,
$$

where $c$ stands for the leading coefficient in (5.45). According to Definition 6.1 and (5.39), the square bracket converges in $\mathcal{S}^{\prime}(\mathbb{R})$, and therefore (6.23) makes sense in the limit $\varepsilon \searrow 0$ if we interpret the round brackets as the test function.

To analyze (4.12), we split up the integral into an integral over the interval $a \in(0,1]$ plus an integral over $[1, \infty)$. Using the explicit form of $\hat{\mathcal{N}}_{\varepsilon}$, the integral over $(0,1]$ can again be written in the form $(6.23)$, which establishes convergence as $\varepsilon \searrow 0$. The integral over $[1, \infty)$, on the other hand, can be analyzed exactly as that in (4.7), because $\hat{\mathcal{N}}^{\varepsilon}$ and $\hat{\mathcal{M}}^{\varepsilon}$ coincide for large $a$ according to (4.9) and (5.44).

\section{Additional free parameters}

In view of the application to state stability, the following consideration gives rise to additional free parameters. As is shown in $[5$, Appendix A], it is possible to regularize the fermionic projector in such a way that the property of a distribution $\mathcal{M} P$-product is no longer satisfied, but where the continuum limit yields an additional distributional contributions supported at $\xi=0$. More precisely, (4.21) is replaced by

$$
Q(\xi)=\frac{1}{2} \tilde{\mathcal{M}}(\xi) P(\xi)+c_{2} \delta^{4}(\xi)-c_{3} i \not \partial \delta^{4}(\xi)-c_{4} \square \delta(\xi)
$$

with real parameters $c_{2}, c_{3}$ and $c_{4}$, which can be chosen arbitrarily. Let us consider how these additional parameters can be built into the variational principle of Definition 2.1. Taking the Fourier transform, we see that the additional terms change the functions $a$ and $b$ in representation (4.22) by

$$
a\left(k^{2}\right) \rightarrow a\left(k^{2}\right)+c_{3}|k|, \quad b\left(k^{2}\right) \rightarrow b\left(k^{2}\right)+c_{2}+c_{1} k^{2}
$$

The parameter $c_{2}$ changes $Q$ only by an irrelevant constant, and thus we may disregard this parameter. Then, going through the calculation in the proof of Theorem 4.1, one immediately verifies that the terms in (7.1) can be arranged by modifying the action as follows. 
Definition 7.1. We define the extended action $\mathcal{S}_{\text {ext }}$ by

$$
\mathcal{S}_{\text {ext }}=\mathcal{S}+c_{3} \sum_{\beta=1}^{g} \rho_{\beta} m_{\beta}^{4}+c_{4} \sum_{\beta=1}^{g} \rho_{\beta} m_{\beta}^{5}
$$

with $\mathcal{S}$ as in (2.6). Here $c_{3}$ and $c_{4}$ are two free real parameters. The corresponding variational principle with constraint (2.13) is referred to as the extended variational principle.

\section{Numerical construction of minimizers}

We first rewrite the action in a form which is most convenient for the numerical analysis.

Proposition 8.1. (Extended action in momentum space). We introduce the real-valued function

$$
H(a, x, y)=\frac{1}{a}(J(a, x, y)+K(a, x, y))
$$

(with the functions $J$ and $K$ as given by (5.36), (5.40)) and choose a parameter

$$
a_{\max }>\max _{\beta \in\{1, \ldots, g\}} m_{\beta}^{2} .
$$

Then the extended action (see Definitions 7.1 and 2.1) can be written as

$$
\begin{aligned}
\mathcal{S}_{\text {ext }}= & \frac{1}{2^{16} \pi^{10}} \sum_{\alpha, \beta, \gamma, \delta} \rho_{\alpha} \rho_{\beta} \rho_{\gamma} \rho_{\delta} \int_{0}^{a_{\max }} H\left(a, m_{\alpha}, m_{\beta}\right) H\left(a, m_{\gamma}, m_{\delta}\right) d a \\
& +F\left(a_{\max }, \mathfrak{m}_{3}, \mathfrak{m}_{5}\right)+c_{3} \sum_{\beta=1}^{g} \rho_{\beta} m_{\beta}^{4}+c_{4} \sum_{\beta=1}^{g} \rho_{\beta} m_{\beta}^{5} .
\end{aligned}
$$

Proof. It clearly suffices to consider $\mathcal{S}$, because the extended action is obtained simply by adding the extra terms in Definition 7.1. From (6.21) and (5.47) it is clear that $\hat{\mathcal{M}}^{\varepsilon}(k) \stackrel{\varepsilon \rightarrow 0}{\rightarrow} \hat{\mathcal{M}}(k)$ uniformly on the set $\{k \mid 0<$ $\left.k^{2}<a_{\max }\right\}$, whereas for large $k^{2}, \hat{\mathcal{M}}^{\varepsilon}$ is given explicitly by

$$
\hat{\mathcal{M}}^{\varepsilon}(k)=2 \pi^{2} \not k \Theta\left(k^{2}\right) \epsilon\left(k^{0}\right)\left(\frac{\mathfrak{m}_{3}}{k^{2}} e^{-\frac{\varepsilon k^{2}}{2}}+\frac{4 \mathfrak{m}_{5}}{k^{4}}\right) \quad \text { if } k^{2}>a_{\max } .
$$


Hence, using the result of Theorem 5.2 in the formula for the regularized action in momentum space (4.7), we obtain

$$
\begin{aligned}
\mathcal{S}= & \frac{1}{2^{16} \pi^{10}} \sum_{\alpha, \beta, \gamma, \delta} \rho_{\alpha} \rho_{\beta} \rho_{\gamma} \rho_{\delta} \int_{0}^{a_{\max }} H\left(a, m_{\alpha}, m_{\beta}\right) H\left(a, m_{\gamma}, m_{\delta}\right) d a \\
& +\frac{1}{(2 \pi)^{4}} \lim _{\varepsilon \searrow 0}\left(4 \pi^{4} \int_{a_{\max }}^{\infty}\left(\mathfrak{m}_{3} \mathrm{e}^{-\frac{\varepsilon k^{2}}{2}}+4 \mathfrak{m}_{5} a^{-1}\right)^{2} d a+F_{\varepsilon}\left(\mathfrak{m}_{3}, \mathfrak{m}_{5}\right)\right) .
\end{aligned}
$$

The result follows because the last line depends only on $a_{\max }, \mathfrak{m}_{3}$ and $\mathfrak{m}_{5}$.

Note that the argument before (5.46) shows that the function $H,(8.1)$, is bounded near $a=0$. Hence in (8.2) we only need to integrate a bounded continuous function over a compact interval. This makes it possible to compute the action with standard numerical methods. Using standard tools of nonlinear optimization, one can construct numerical minimizers.

Alternatively, one can construct critical points by searching for numerical solutions of conditions (a) and (b) in Theorem 4.1. It is helpful that the resulting equations are polynomials in the parameters $\rho_{\beta}$, making it possible to use specific algorithms for computing the roots of systems of polynomials. For a detailed description of the numerics we refer to the $\mathrm{PhD}$ thesis [8]; here we proceed by discussing a few of the numerical solutions.

In the case $g=1$ of one generation, we constructed all minimizers of the unextended action (i.e., $c_{3}=c=4=0$ ). By scaling we can assume that $m_{1}=1$. We found a one-parameter family of minimizers parametrized by $c_{1}$, whereas $c_{0}$ must vanish. In figure 6 the variation density is shown in typical examples. In agreement with Theorems 4.1 and 4.2 , we see that $V^{\prime}(1)=0$. However, the solutions are not state stable, because condition (iii) in Definition 4.1 (or equivalently condition (iii') on page 27) is violated. More specifically, the variation density has near $m=1$ the expansion

$$
V(m)=(m-1)^{2}\left(\alpha c_{1}+\beta \log |m-1|\right)+o\left((m-1)^{2}\right)
$$

with constants $\alpha, \beta>0$. Therefore, it is impossible to arrange that $m=1$ is a local minimum of $V$. Since the extra contributions of the extended action are polynomials in $m$, this statement remains true for the extended action. We conclude that there is no state-stable Dirac sea configuration with one generation. In words, our action principle prefers to "split up" the Dirac sea into two seas. However, by choosing $c_{1}$ sufficiently large we can arrange that the minima of $V$ are arbitrarily close to $m=1$ (see figure 6 right). 

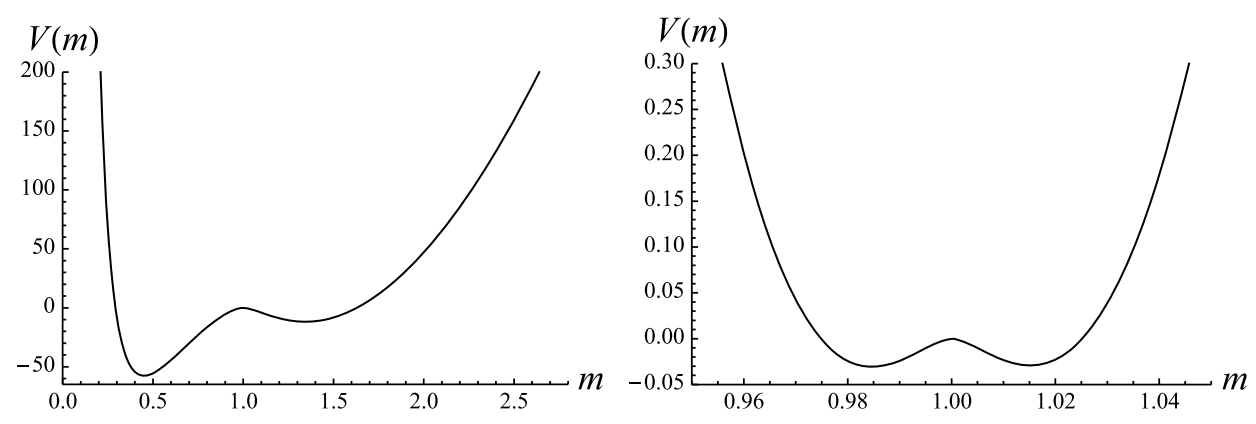

Figure 6: The variation density in the case of one generation of mass $m_{1}=$ 1 and the parameters $c_{0}=c_{3}=c_{4}=0$ and $c_{1}=2 \times 10^{6}$ (left), $c_{1}=10^{7}$ (right).

The case $g=2$ of two generations gives some insight into the nature of our variational principle. By scaling we can always assume that $m_{1}=$ $1<m_{2}$ and $\rho_{1}=1$. Thus for the unextended action we have the four free parameters $m_{2}, \rho_{2}, c_{0}$ and $c_{1}$ to satisfy the three conditions $V^{\prime}\left(m_{1}\right)=0=$ $V^{\prime}\left(m_{2}\right)$ and $V\left(m_{1}\right)=V\left(m_{2}\right)$. Therefore, one expects for given $m_{2}$ a discrete number of minima. Indeed, we found for every $m_{2}$ exactly one minimizer. In figure 7 the variation density of the minimizers is shown for different values of $m_{2}$. If $m_{2}$ is close to $m_{1}$, the result can be understood in analogy to the minimizers for one generation: again the points $m=m_{j}$ are local maxima of the variation density. Thus the solution is not state stable, and our action principle has the tendency to split up the two Dirac seas into even more seas. However, if $m_{2}$ is increased, a "potential wall" emerges between $m_{1}$ and $m_{2}$, such that the number of local minima of $V$ increases from three to four (see the cases $m_{2}=4.25$ and 4.5). The points $m=m_{j}$ are still local maxima, but the neighboring local minima are very close (see the case $m_{2}=5$ ). If $m_{2}$ is further increased, the local minima approach the points $m=m_{j}$ exponentially fast, and thus the resulting variation density looks very much like a state stable configuration (see the case $m_{2}=10$ ). Keeping in mind that the physical masses of the three generations of leptons scale with factors of the order 100, and that a relative uncertainty in the rest masses of $10^{-100}$ can certainly not be measured in experiments, in such cases it is fair to identify the points $m_{j}$ with the neighboring minima, so that we may regard the $m_{j}$ as absolute minima of $V$.

In the case $g=3$ of three generations, we can assume by scaling that $1=$ $m_{1}<m_{2}<m_{3}$. We found different classes of minimizers. In view of the physical masses of the leptons, we were most interested in constructing solutions where the masses are far apart, i.e. $m_{1} \ll m_{2} \ll m_{3}$. Our attempts to construct such solutions were successful only for the extended action. Figure 8 gives an example of a state stable Dirac sea configuration with 

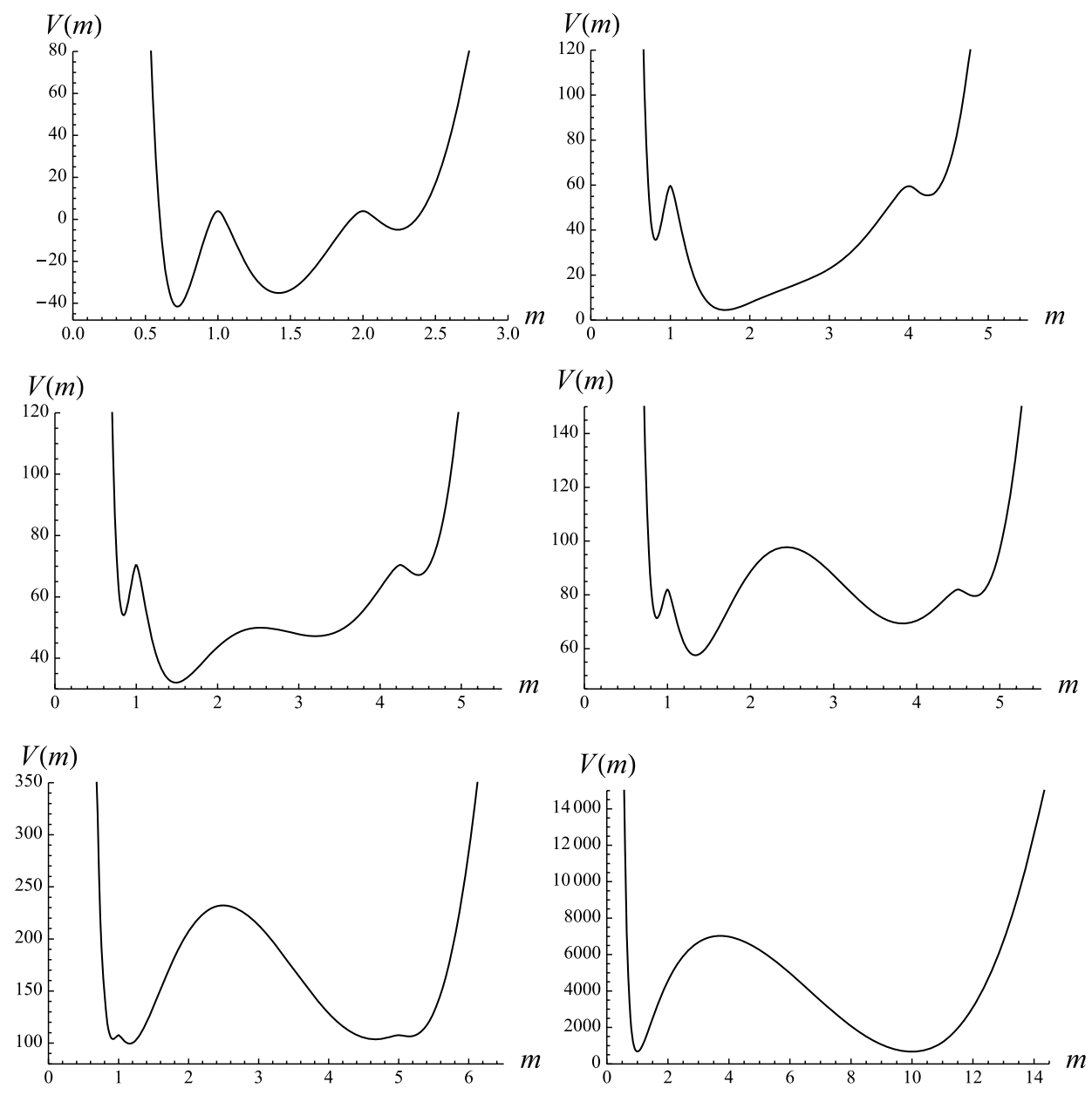

Figure 7: Minimizers of the unextended action in the case of two generations of masses $m_{1}=1$ and $m_{2}=2,4,4.25,4.5,5$ and 10 .

three generations. The corresponding values of the parameters are $m_{2}=$ $5, m_{3}=20, \rho_{1}=1, \rho_{2}=10^{-4}, \rho_{3}=9.696 \times 10^{-6}, c_{0}=-6.692 \times 10^{8}, c_{1}=$ $-2.516 \times 10^{9}, c_{3}=9658.25$ and $c_{4}=8416.56$.

As already mentioned in the introduction, the systematic study of the minimizers goes beyond the scope of this paper. Clarifying the detailed structure of the minimizers and critical points of our action is an important project for the future. Talking of future developments, we finally point out that information on the constants $c_{0}, c_{1}, c_{2}$ and $c_{4}$ could be obtained by studying regularized or discretized Dirac sea systems (for example using numerical methods [7] for large systems). Moreover, the weight factors $\rho_{\beta}$ have an influence on the continuum limit (see [5, Appendix A]), and thus 

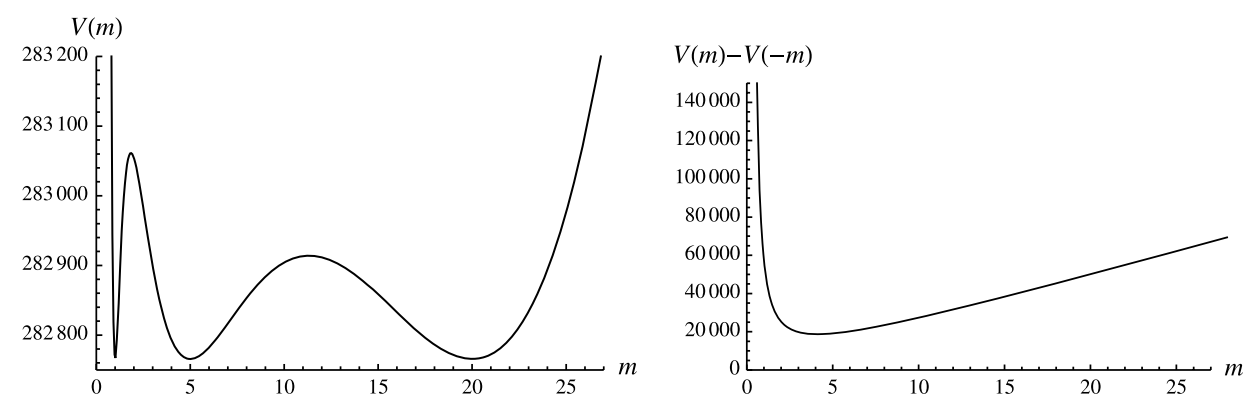

Figure 8: A state stable Dirac sea structure with three generations.

the detailed analysis of the continuum limit for interacting systems should give constraints for the weight factors. In view of these future investigations, which should reduce our number of free parameters, the introduced action principle seems to be a promising step towards a physical theory which makes predictions for the ratios of the masses of elementary particles.

\section{Acknowledgment}

Supported in part by the Deutsche Forschungsgemeinschaft.

\section{References}

[1] F. Finster, The principle of the fermionic projector, AMS/IP Studies in Advanced Mathematics, 35, American Mathematical Society, Providence, RI, 2006, viii+302.

[2] F. Finster, A variational principle in discrete space-time-existence of minimizers, Calc. Var. and Partial Differential Equation 29 (2007), 431-453; math-ph/0503069.

[3] F. Finster, The principle of the fermionic projector: an approach for quantum gravity, gr-qc/0601128, in 'Quantum Gravity', eds. B. Fauser, J. Tolksdorf and E. Zeidler, Birkhäuser Verlag, (2006), 263-281.

[4] F. Finster, Fermion systems in discrete space-time, J. Phys.: Conf. Ser. 67 (2007), 012048; hep-th/0601140.

[5] F. Finster, On the regularized fermionic projector of the vacuum, J. Math. Phys. 49 (2008), 032304 (2007); math-ph/0612003.

[6] F. Finster, From discrete space-time to Minkowski space: Basic mechanisms, methods and perspectives, in 'Recent Developments in Quantum 
Field Theory', eds. B. Fauser, J. Tolksdorf and E. Zeidler, Birkhäuser Verlag, 2009, 235-259; arXiv:0712.0685 [math-ph].

[7] F. Finster and W. Plaum, A lattice model for the fermionic projector in a static and isotropic space-time, Math. Nachr. 281 (2008), 803-816; arXiv:0712.0676 [math-ph].

[8] S. Hoch, State stability analysis of the fermionic projector of the vacuum, Dissertation, Universität Regensburg, 2008.

[9] B. N. Watson, Theory of Bessel functions, 2nd edn., Cambridge University Press, Cambridge, 1958.

[10] A. H. Zemanian, Generalized integral transformations, Pure and Applied Mathematics, xviii, [John Wiley \& Sons, Inc.], New YorkLondon-Sydney, 1968, xvi+300pp. 
